\title{
Scattering Studies with Low-Energy Kaon-Proton Femtoscopy in Proton-Proton Collisions at the LHC
}

\author{
S. Acharya et al. ${ }^{*}$ \\ (A Large Ion Collider Experiment Collaboration)
}

(Received 18 July 2019; revised manuscript received 3 December 2019; accepted 11 February 2020; published 6 March 2020)

\begin{abstract}
The study of the strength and behavior of the antikaon-nucleon $(\bar{K} N)$ interaction constitutes one of the key focuses of the strangeness sector in low-energy quantum chromodynamics (QCD). In this Letter a unique high-precision measurement of the strong interaction between kaons and protons, close and above the kinematic threshold, is presented. The femtoscopic measurements of the correlation function at low pair-frame relative momentum of $\left(K^{+} p \oplus K^{-} \bar{p}\right)$ and $\left(K^{-} p \oplus K^{+} \bar{p}\right)$ pairs measured in $p p$ collisions at $\sqrt{s}=5,7$, and $13 \mathrm{TeV}$ are reported. A structure observed around a relative momentum of $58 \mathrm{MeV} / c$ in the measured correlation function of $\left(K^{-} p \oplus K^{+} \bar{p}\right)$ with a significance of $4.4 \sigma$ constitutes the first experimental evidence for the opening of the $\left(\bar{K}^{0} n \oplus K^{0} \bar{n}\right)$ isospin breaking channel due to the mass difference between charged and neutral kaons. The measured correlation functions have been compared to Jülich and Kyoto models in addition to the Coulomb potential. The high-precision data at low relative momenta presented in this work prove femtoscopy to be a powerful complementary tool to scattering experiments and provide new constraints above the $\bar{K} N$ threshold for low-energy QCD chiral models.
\end{abstract}

DOI: 10.1103/PhysRevLett.124.092301

The kaon $(K)$ nucleon $(N)$ and antikaon $(\bar{K}) N$ interactions constitute the building blocks of low energy QCD with $u, d$, and $s$ quarks, since the effective theories aiming to describe hadron interactions in the nonperturbative energy regime are anchored to these interactions. Traditionally, the interaction of $K$ and $\bar{K}$ with protons and neutrons has been studied by performing scattering experiments at low energies. However, only few measurements exist and only in a limited energy range [1-5]. In such experiments the initial state is fixed, formed by a $K N$ or $\bar{K} N$ pair, and cross sections of elastic and inelastic final states are measured.

These data showed that the $K$ and $\bar{K}$ behavior with nucleons is very different: while the repulsive nature of $K^{+} p$, due to the strong and Coulomb interactions, is well established [6], the strong interacting term of the $K^{-} p$ is instead deeply attractive and characterized by the presence of several coupled channels, i.e., two-particle systems with energy close to the $K^{-} p$ threshold and carrying the same quantum numbers. These coupled-channels contributions are already present in the initial $\bar{K} N$ scattering wave function and hence influence both the inelastic and the elastic processes [7].

\footnotetext{
*Full author list given at the end of the article.

Published by the American Physical Society under the terms of the Creative Commons Attribution 4.0 International license. Further distribution of this work must maintain attribution to the author(s) and the published article's title, journal citation, and DOI.
}

In the $K^{-} p$ system, due to the strangeness $S=-1$ charge of the $\bar{K}$, already two open coupled channels appear below threshold: $\Lambda \pi$ and $\Sigma \pi$. Of particular interest is the coupling to the $\Sigma \pi$ channel since this, along with the attractive nature of the $\bar{K} N$ interaction, leads to the appearance of the $\Lambda(1405)$ resonance just $27 \mathrm{MeV} / c^{2}$ below threshold. Indeed, this resonance is interpreted as the only $\Sigma \pi-\bar{K} N$ molecular state [8-10]. The available theoretical approaches [11-20] are constrained above the $\bar{K} N$ threshold, but since the experimental data are scarce, these constraints are rather loose, resulting in rather significant differences below threshold. Experimental constraints on the $\bar{K} N$ interaction and on the interplay between both $\bar{K} N$ and $\Sigma \pi$ poles are fundamental to reproduce the properties of the $\Lambda(1405)$ [21-25].

Approximately $5 \mathrm{MeV}$ above threshold, the $\bar{K}^{0} n$ channel opens up due to the breaking of the isospin symmetry. The $\bar{K} n-K N$ coupling is also very important to understand the interaction and structure of the $\Lambda(1405)$ and its effect should be visible in the total $K^{-} p$ cross section measured in scattering experiments as a clear cusplike structure for a kaon incident momentum of $p_{\mathrm{lab}}=89 \mathrm{MeV} / c$ [26]. However, this peak has not been experimentally observed yet due to the large uncertainties of the data $[3,5,27]$.

In order to constrain the contributions of the coupled channels and to provide a complete description of the $\bar{K} N$ interaction, precise data close to threshold are needed and the effects of coupled channels lying close to threshold must be explicitly taken into account in any process between a $\bar{K}$ and a nucleon. 
The measurement of kaonic hydrogen [28], which nowadays constitutes the most precise constraint at threshold, and the obtained results on the $\bar{K} N$ scattering parameters include the coupled-channel contributions only in an effective way.

Recently, the femtoscopy technique [29,30], which measures the correlation of particle pairs at low relative momentum, has provided high precision data on different baryon-baryon pairs [31-33], indicating a great sensitivity to the underlying strong potential. Contrary to the scattering, in femtoscopy only the final state is measured and different initial states are allowed. In the $K^{-} p$ system, this translates into an extreme sensitivity of the correlation function to the introduction of the different coupledchannels, which affect both shape and magnitude of the femtoscopic signal [34].

The femtoscopic measurement of $K p$ pairs $\left[\left(K^{+} p \oplus\right.\right.$ $\left.K^{-} \bar{p}\right)$ and $\left.\left(K^{-} p \oplus K^{+} \bar{p}\right)\right]$ from $p p$ collisions at different energies presented in this Letter shows experimentally for the first time the impact of the coupled-channels effect on the momentum correlation function. Comparison with recent models including or partially including coupledchannel contributions are presented. The same-charge pairs $\left(K^{+} p \oplus K^{-} \bar{p}\right)$, because of the well-described interaction and the lack of coupled-channel effects, are used as a benchmark to test the sensitivity of the correlation function to the strong interaction.

The analysis presented here is based on minimum bias triggered $p p$ collisions collected by the ALICE experiment [35] at the LHC in 2010, 2015, 2016, and 2017 at three different collision energies $(\sqrt{s}=5,7$, and $13 \mathrm{TeV})$. The correlation function $C\left(k^{*}\right)$ is measured as a function of the momentum difference of the pair $k^{*}=\frac{1}{2}\left(\vec{p}_{1}{ }^{*}-\vec{p}_{2}{ }^{*}\right)$, where $\vec{p}_{1}{ }^{*}$ and $\vec{p}_{2}{ }^{*}$ are the momenta of the two particles in the pair rest frame. It is defined as $C\left(k^{*}\right)=\mathcal{N} A\left(k^{*}\right) / B\left(k^{*}\right)$, where $A\left(k^{*}\right)$ is the measured distribution of pairs from the same event, $B\left(k^{*}\right)$ is the reference distribution of pairs from mixed events, and $\mathcal{N}$ is a normalization parameter. The denominator $B\left(k^{*}\right)$ is formed by mixing particles from one event with particles from a pool of other events with a comparable number of charged particles at midrapidity [36] and a comparable interval of the collision primary vertex coordinate along the beam axis, $V_{z}$ interval $\left(\Delta V_{z}=2 \mathrm{~cm}\right)$. The normalization parameter $\mathcal{N}$ is chosen such that the mean value of the correlation function equals unity for $400<k^{*}<600 \mathrm{MeV} / c$.

The main subdetectors used in this analysis are the V0 detectors [37], which are used as trigger detectors, the inner tracking system (ITS) [38], the time projection chamber (TPC) [39] and the time-of-flight (TOF) detector [40]. The ITS, TPC, and TOF are located inside a $0.5 \mathrm{~T}$ solenoidal magnetic field and are used to track and identify charged particles. In order to ensure a uniform acceptance at midrapidity, events were selected by requiring the $V_{z}$ of the event to be within $10 \mathrm{~cm}$ from the center of the ALICE detector. The rejection of pileup is performed by exploiting the innermost silicon detector (SPD, part of ITS) vertexing capabilities, following the same procedure described in Refs. [33,41]. After the application of the event selection criteria, about 874 million, 374 million, and 1 billion minimum bias $p p$ events were analyzed at $\sqrt{s}=5,7$, and $13 \mathrm{TeV}$, respectively.

As recently proposed in Ref. [42], in order to reduce the contribution from the minijet background in $p p$ collisions, the events were classified according to their transverse sphericity $\left(S_{T}\right)$, an observable which is known to be correlated with the number of hard parton-parton interactions in each event [43]. An event with only one hard parton-parton interaction will generally produce a jetlike distribution that yields low sphericity, while an event with several independent hard parton-parton interactions can yield higher sphericity. To reduce the strong minijet background at low momenta, only events with $S_{T}$, defined as in Ref. [42], larger than 0.7 were considered in this analysis.

Charged particles were tracked and selected using the same criteria described in Ref. [33]. The charged kaons and protons were identified in a wide transverse momentum $\left(p_{T}\right)$ interval $\left(0.15<p_{T}<1.4 \mathrm{GeV} / c\right.$ for kaons and $0.4<$ $p_{T}<3 \mathrm{GeV} / c$ for protons) using the information provided by the TPC and the TOF detectors. The deviation of the measured specific ionization energy loss $(d E / d x)$ in the TPC from the Bethe-Bloch parametrization was required to be within 3 standard deviations $\left(\sigma_{\mathrm{TPC}}\right)$. For kaons with $p_{T}>$ $0.4 \mathrm{GeV} / c$ and protons with $p_{T}>0.8 \mathrm{GeV} / c$, a similar method was applied for the particle identification using the TOF, where, on top of TPC selection, a selection based on a maximum 3 standard deviation difference from the expected signal at a given momentum was applied. Tracks identified ambiguously as belonging to both a proton and a kaon were discarded. In order to remove the large fraction of $e^{+} e^{-}$pairs that can affect the extraction of the correlation function of the opposite-charge pairs, a selection on the $p_{T}$ of kaon and protons was applied: kaon candidates are excluded if $0.3<$ $p_{T}<0.4 \mathrm{GeV} / c$, while proton candidates are excluded in the interval between $0.6<p_{T}<0.8 \mathrm{GeV} / c$. The purity of the selected particle samples, determined by Monte Carlo simulations, is larger than $99 \%$ in the considered $p_{T}$ intervals for all the analyzed dataset. The systematic uncertainties of the measured $C\left(k^{*}\right)$ were evaluated for each $k^{*}$ interval by varying event and track selection criteria. The event sample was varied by changing the selection on the $V_{z}$ position from \pm 10 to $\pm 7 \mathrm{~cm}$ and by varying the sphericity of the accepted events from $S_{T}>0.7$ to $S_{T}>0.6$ and $S_{T}>0.8$. Systematic uncertainties related to the track selection criteria were studied by varying the selection on the distance of closest approach in the transverse plane direction within the experimental resolution. To study systematic effects related to particle identification, the number of standard deviations around the energy loss expected for kaons and protons in the TPC and, similarly, for the time of 
flight in the TOF was modified from $3 \sigma$ to $2 \sigma$. For each source, the systematic uncertainty was estimated as the root mean square (RMS) of the deviations. The total systematic uncertainty was calculated as the quadratic sum of each source's contribution and amounts to about 3\% in the considered $k^{*}$ intervals.

The measured correlation functions for $\left(K^{+} p \oplus K^{-} \bar{p}\right)$ and $\left(K^{-} p \oplus K^{+} \bar{p}\right)$ are shown in the upper panels of Figs. 1 and 2. In both figures, each panel corresponds to a different collision energy, as indicated in the legend. The structure that can be seen in the $\left(K^{-} p \oplus K^{+} \bar{p}\right)$ correlation function at $k^{*}$ around $240 \mathrm{MeV} / c$ in Fig. 2 is consistent with the $\Lambda(1520)$ which decays into $K^{-} p$, with a center-of-mass momentum for the particle pair of $243 \mathrm{MeV} / c$ [44]. The correlation function of $\left(K^{-} p \oplus K^{+} \bar{p}\right)$ exhibits also a clear structure between 50 and $60 \mathrm{MeV} / c$ for the three collision energies. The $k^{*}$ position of the structure is consistent with the threshold of the $\bar{K}^{0} n\left(K^{0} \bar{n}\right)$ channel opening at $p_{\text {lab }}=$ $89 \mathrm{MeV} / c[3,5,27]$ which corresponds to $k^{*}=58 \mathrm{MeV} / c$. In order to quantify the significance of this structure, and since the three measured distributions are mutually compatible, the $C\left(k^{*}\right)$ measured at the three different energies were summed using the number of pairs in each data sample as a weight. The resulting $C\left(k^{*}\right)$ was interpolated with a spline considering the statistical uncertainties and the derivative of the spline was then evaluated [36]. A change in the slope of the derivative consistent with a cusp effect in the $k^{*}$ region between 50 and $60 \mathrm{MeV} / c$ at the level of $4.4 \sigma$ has been observed, to be compared with a significance of $30 \sigma$ for $\Lambda(1520)$. The measurement presented here is therefore the first experimental evidence for the opening of the $\bar{K}^{0} n\left(K^{0} \bar{n}\right)$ channel, showing that the femtoscopy technique is a unique tool to study the $\bar{K} p$ interaction and coupled-channel effects.
The experimental correlation functions were also used to test different potentials to describe the interaction between $K^{+} p\left(K^{-} \bar{p}\right)$ and $K^{-} p\left(K^{+} \bar{p}\right)$. The measured correlation function $C\left(k^{*}\right)$ is compared with a theoretical function using the following equation

$C\left(k^{*}\right)=\left(a+b \cdot k^{*}\right) \cdot\left\{1+\lambda \cdot\left[C\left(k^{*}\right)^{\text {theoretical }}-1\right]\right\}$,

where the baseline $\left(a+b \cdot k^{*}\right)$ is introduced to take into account the remaining nonfemtoscopic background contributions related to momentum-energy conservation which might be present also after the $S_{T}$ selection. The slope $b$ of the baseline is fixed from Monte Carlo simulations based on PYTHIA 6 [45] and PYTHIA 8 [46], while the normalization $a$ is a free parameter. In order to assign a systematic uncertainty related to the slope of the baseline, the $b$ parameter has been varied by its uncertainty as obtained from the Monte Carlo simulation $( \pm 10 \%)$ and the fit repeated. The parameter $\lambda$ represents the fraction of primary pairs in the analyzed sample multiplied by the purity of the same sample and is fixed by fitting Monte Carlo (MC) templates to the experimental distributions of $\mathrm{DCA}_{x y}$ of kaons and protons, similarly to what is described in Ref. [33].

The model correlation function, $C\left(k^{*}\right)^{\text {theoretical }}$, is evaluated using the CATS framework [47]. The $\lambda$ parameters obtained for each analyzed dataset are reported in each panel of Figs. 1 and 2 for same-charge and opposite-charge Kp pairs, and vary from 0.61 to 0.76 for each considered set. A systematic uncertainty of $\pm 10 \%$ is associated with the $\lambda$ parameters. This uncertainty was estimated by varying the Monte Carlo templates used in the feed-down estimation procedure based on PYTHIA 6 [45] for the analysis at $\sqrt{s}=7 \mathrm{TeV}$ and based on PYTHIA 8 [46] for the analyses performed at $\sqrt{s}=5$ and $13 \mathrm{TeV}$, and varying

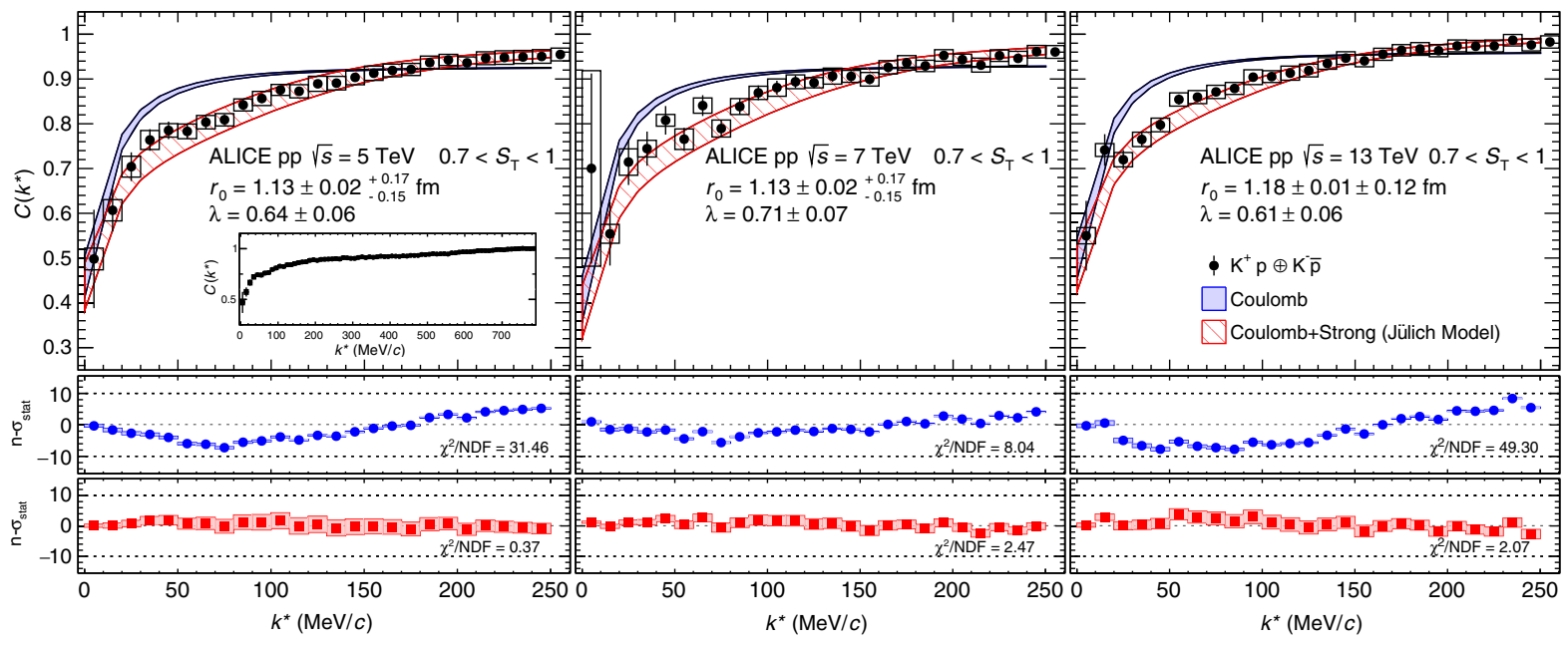

FIG. 1. $\left(K^{+} p \oplus K^{-} \bar{p}\right)$ correlation functions obtained from $p p$ collisions at $\sqrt{s}=5$ (left), 7 (middle), and 13 TeV (right). The inset shows the correlation function evaluated for $p p$ collisions at $\sqrt{s}=5 \mathrm{TeV}$ in a wider $k^{*}$ interval. The measurement is shown by the black markers; the vertical lines and the boxes represent the statistical and systematic uncertainties, respectively. Bottom panels represent comparison with models as described in the text. 


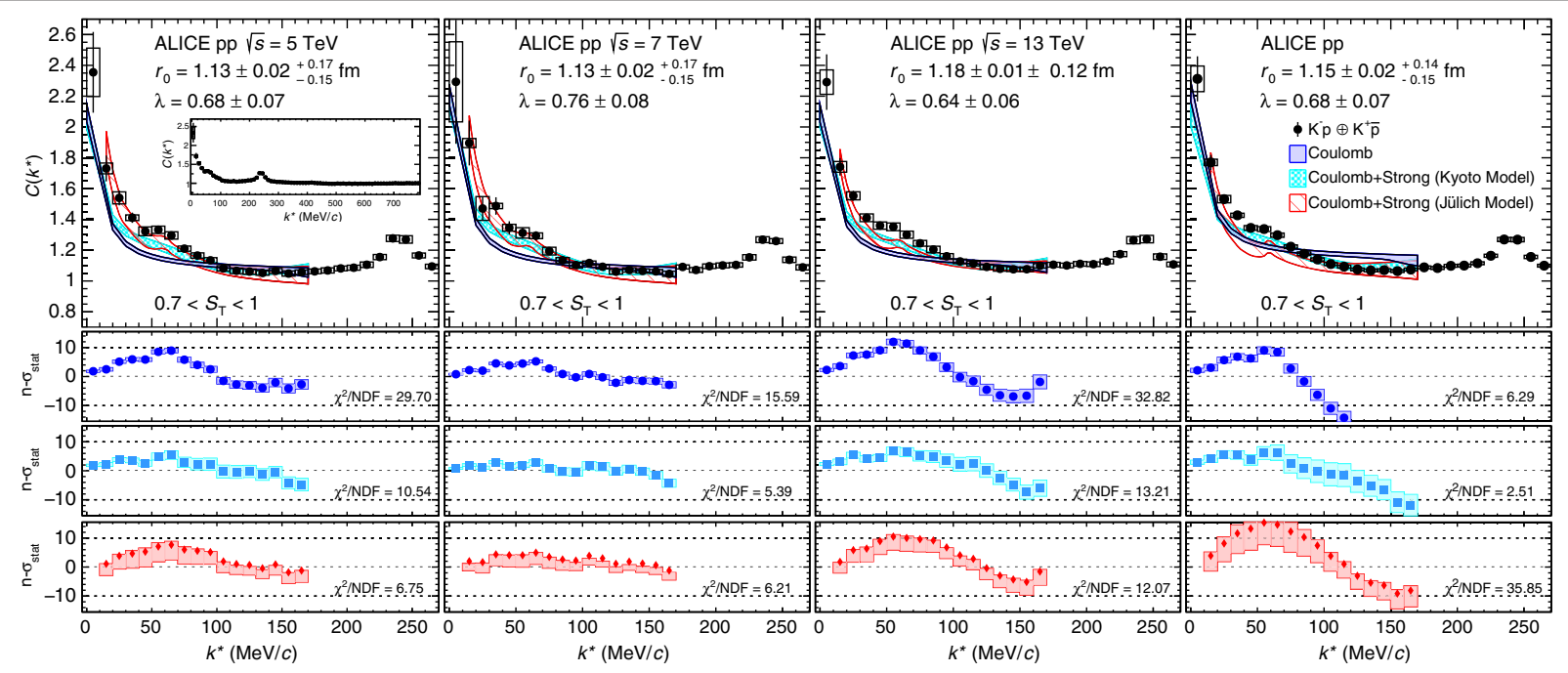

FIG. 2. $\left(K^{-} p \oplus K^{+} \bar{p}\right)$ correlation functions obtained (from left to right) from $p p$ collisions at $\sqrt{s}=5,7,13 \mathrm{TeV}$. The fourth panel shows the combined results at the three colliding energies; the number of pairs in each data sample has been used as weight. The inset shows the correlation function evaluated for $p p$ collisions at $\sqrt{s}=5 \mathrm{TeV}$ in a wider $k^{*}$ interval. The measurement is presented by the black markers; the vertical lines and the boxes represent the statistical and systematic uncertainties, respectively. Bottom panels represent comparison with models as described in the text.

the transport code used in the simulation from GEANT3 [48] to GEANT4 [49].

The effects related to momentum resolution effects are accounted for by correcting the theoretical correlation function, similarly to what shown in Refs. [33] and [41]. The theoretical correlation function $C\left(k^{*}\right)^{\text {theoretical }}$ depends not only on the interaction between particles, but also on the profile and the size of the particle emitting source. Under the assumption that there is a common Gaussian source for all particle pairs produced in $p p$ collisions at a fixed energy, the size of the source considered in the present analysis is fixed from the baryon-baryon analyses described in Refs. [33] and [41]. The impact of strongly decaying resonances (mainly $K^{*}$ decaying into $K$ and $\Delta$ decaying into $p$ ) on the determination of the radius for $K p$ pairs was studied using different Monte Carlo simulations [45,46] and found to be $10 \%$. This contribution was linearly added to the systematic uncertainty associated with the radius. The radii of the considered Gaussian sources are $r_{0}=$ $1.13 \pm 0.02_{-0.15}^{+0.17} \mathrm{fm}$ [33] for collisions at $\sqrt{s}=5$ and $7 \mathrm{TeV}$, and $r_{0}=1.18 \pm 0.01 \pm 0.12 \mathrm{fm}$ [41] for the $\sqrt{s}=$ $13 \mathrm{TeV}$ collisions.

The comparison of the measured $C\left(k^{*}\right)$ for same-charge $K p$ pairs with different models is shown in Fig. 1. Each panel presents the results at different collision energy and the comparison with two different scenarios. The blue band represents the correlation function evaluated as described in Eq. (1), assuming only the presence of the Coulomb potential to evaluate the $C\left(k^{*}\right)^{\text {theoretical }}$ term. The red band represents the correlation function assuming the strong potential implemented in the Jülich model [50] in addition to the Coulomb potential. The latter has been implemented using the Gamow factor [51]. In the bottom panels, the difference between data and model evaluated in the middle of each $k^{*}$ interval, and divided by statistical error of data for the three considered collision energies are shown. The width of the bands represents the $n-\sigma$ range associated to the model variations. The reduced $\chi^{2}$ are also shown. This comparison reveals that the Coulomb interaction is not able to describe the data points, as expected, while the introduction of a strong potential allows us to reproduce consistently the data when the same source radius as for baryon-baryon pairs is considered. Hence, the measured correlation functions are sensitive to the strong interaction and can be used to test different strong potentials for the $K^{-} p$ system, assuming a common source for all the $K p$ pairs produced in a collision.

Similar to Fig. 1 for like-sign pairs, Fig. 2 shows the data-model comparison for unlike-sign pairs. The measured $C\left(k^{*}\right)$ is reported for the three different collision energies and the $C\left(k^{*}\right)$ distributions were compared with different interaction models. Since all the models considered in this Letter do not take the presence of $\Lambda(1520)$ into account, only the region below $170 \mathrm{MeV} / c$ is considered in the comparison. The blue bands show results obtained using CATS with a Coulomb potential only.

The remaining curves include, on top of the Coulomb attraction, different descriptions of the $\bar{K} N$ strong interaction. The width of each band accounts for the uncertainties in the $\lambda$ parameters, the source radius and the baseline. The light blue bands corresponds to the Kyoto model calculations with approximate boundary conditions on the $K^{-} p$ wave function which neglect the contributions from $\Sigma \pi$ and $\Lambda \pi$ coupled channels [26,52-55]. Moreover, 
this version of the Kyoto model is performed in the socalled isospin basis and hence does not include the mass difference between $K^{-}$and $\bar{K}^{0}$ : no cusplike structure are foreseen by the model in $C\left(k^{*}\right)$.

The introduction of coupled-channel contributions in the correlation function has been shown to result in additional attractive terms enhancing the signal, in particular in the low $k^{*}$ region [34]. As expected, the Kyoto results clearly underestimate the data at low momenta where the $\Sigma \pi$ channel is particularly relevant.

The red bands indicate results obtained with the Jülich strong potential, recently updated to reproduce the kaonic atom results from SIDDHARTA collaboration [34]. This model includes explicitly both $\Sigma \pi$ and $\Lambda \pi$ coupled channels below threshold and the $K^{-}-\bar{K}^{0}$ mass difference, reflected in the presence of a cusp structure. Accordingly, the comparison with data shows a better agreement with respect to the Kyoto model, but the region of $k^{*}$ below $100 \mathrm{MeV} / c$ is nevertheless not fully reproduced and the shape of the correlation function deviates from the data around the cusp.

The overall tension between data and the models is not surprising since the latter were fitted to only reproduce scattering data above threshold (providing constraints for $k^{*} \geq 70 \mathrm{MeV} / c$ ) and the SIDDHARTA results at threshold [28].

To test the stability of the results, the measured $C\left(k^{*}\right)$ without any $S_{T}$ cut was used and the background from minijets and other kinetically correlated pairs was subtracted by using a Monte Carlo simulation based on PYTHIA 8 [46], using a procedure similar to the one described in Ref. [56]. Applying this method the comparison between data and models is consistent within statistical uncertainties with the one obtained using the sphericity selection.

To summarize, the momentum dependent correlations of same-charge and opposite-charge $K p$ pairs $\left[\left(K^{+} p \oplus K^{-} \bar{p}\right)\right.$ and $\left.\left(K^{-} p \oplus K^{+} \bar{p}\right)\right]$ were measured using the two-particle correlation function in $p p$ collisions at different collision energies. A structure around $k^{*}=58 \mathrm{MeV} / c$ in the measured correlation function of $\left(K^{-} p \oplus K^{+} \bar{p}\right)$ was observed. The significance of such a structure was evaluated by combining the results from the three analyzed datasets and by interpolating the total correlation function with a spline. By studying the variation in the slope of the derivative of such a spline in the range $50 \leq k^{*} \leq 60 \mathrm{MeV} / c$, the kinematic cusp was assessed at a $4.4 \sigma$ level. The observed structure is consistent with the opening of the $\bar{K}^{0} n$ channel $\left(p_{\text {lab }} \sim 89 \mathrm{MeV} / c\right)$. This measurement represents the first experimental evidence for the $\bar{K}^{0} n\left(K^{0} \bar{n}\right)$ isospin breaking coupled channel and shows experimentally the effect of coupled-channel contributions on the correlation function.

The measured $C\left(k^{*}\right)$ were compared to different interaction scenarios. The $\left(K^{+} p \oplus K^{-} \bar{p}\right)$ correlation functions were proven to be sensitive to the strong interaction, since a Coulomb-only hypothesis is insufficient to describe the data. The inclusion of the strong interaction via the Jülich model results in a reasonable description of the data within uncertainties. The $\left(K^{-} p \oplus K^{+} \bar{p}\right)$ correlation functions at low $k^{*}$ cannot be fully reproduced by the considered potentials. Nevertheless, model including explicitly coupled-channel contributions shows a better agreement with data. The data presented here represent the most precise experimental information for the $K N$ interaction and provide new constraints for future low-energy phenomenological QCD calculations that can be used to shed light on the nature of the $\bar{K} N$ interaction.

The ALICE Collaboration is grateful to Professor Tetsuo Hyodo and Professor Johann Haidenbauer for the valuable suggestions and discussions. The ALICE Collaboration would like to thank all its engineers and technicians for their invaluable contributions to the construction of the experiment and the CERN accelerator teams for the outstanding performance of the LHC complex. The ALICE Collaboration gratefully acknowledges the resources and support provided by all Grid centres and the Worldwide LHC Computing Grid (WLCG) Collaboration. The ALICE Collaboration acknowledges the following funding agencies for their support in building and running the ALICE detector: the A. I. Alikhanyan National Science Laboratory (Yerevan Physics Institute) Foundation (ANSL) and the State Committee of Science and World Federation of Scientists (WFS), Armenia; the Austrian Academy of Sciences, Austrian Science Fund (FWF): [M 2467-N36] and Nationalstiftung für Forschung, Technologie und Entwicklung, Austria; the Ministry of Communications and High Technologies, National Nuclear Research Center, Azerbaijan; the Conselho Nacional de Desenvolvimento Científico e Tecnológico (CNPq), Universidade Federal do Rio Grande do Sul (UFRGS), Financiadora de Estudos e Projetos (Finep) and Fundação de Amparo à Pesquisa do Estado de São Paulo (FAPESP), Brazil; the Ministry of Science \& Technology of China (MSTC), the National Natural Science Foundation of China (NSFC), and the Ministry of Education of China (MOEC), China; the Croatian Science Foundation and Ministry of Science and Education, Croatia; the Centro de Aplicaciones Tecnológicas y Desarrollo Nuclear (CEADEN), Cubaenergía, Cuba; the Ministry of Education, Youth and Sports of the Czech Republic, Czech Republic; The Danish Council for Independent Research I Natural Sciences, the Carlsberg Foundation and the Danish National Research Foundation (DNRF), Denmark; the Helsinki Institute of Physics (HIP), Finland; the Commissariat à l'Energie Atomique (CEA), the Institut National de Physique Nucléaire et de Physique des Particules (IN2P3), and the Centre National de la Recherche Scientifique (CNRS) and Région des Pays de la Loire, France; Bundesministerium für Bildung und Forschung (BMBF) and GSI Helmholtzzentrum für Schwerionenforschung GmbH, Germany; the General 
Secretariat for Research and Technology, Ministry of Education, Research and Religions, Greece; the National Research, Development and Innovation Office, Hungary; the Department of Atomic Energy Government of India (DAE), the Department of Science and Technology, Government of India (DST), University Grants Commission, Government of India (UGC), and the Council of Scientific and Industrial Research (CSIR), India; the Indonesian Institute of Science, Indonesia; Centro Fermi-Museo Storico della Fisica e Centro Studi e Ricerche Enrico Fermi and Istituto Nazionale di Fisica Nucleare (INFN), Italy; the Institute for Innovative Science and Technology, the Nagasaki Institute of Applied Science (IIST), Japan Society for the Promotion of Science (JSPS) KAKENHI, and the Japanese Ministry of Education, Culture, Sports, Science and Technology (MEXT), Japan; Consejo Nacional de Ciencia (CONACYT) y Tecnología, through Fondo de Cooperación Internacional en Ciencia y Tecnología (FONCICYT) and Dirección General de Asuntos del Personal Academico (DGAPA), Mexico; Nederlandse Organisatie voor Wetenschappelijk Onderzoek (NWO), Netherlands; The Research Council of Norway, Norway; Commission on Science and Technology for Sustainable Development in the South (COMSATS), Pakistan; Pontificia Universidad Católica del Perú, Peru; the Ministry of Science and Higher Education and National Science Centre, Poland; Korea Institute of Science and Technology Information and National Research Foundation of Korea (NRF), Republic of Korea; the Ministry of Education and Scientific Research, Institute of Atomic Physics and the Ministry of Research and Innovation and Institute of Atomic Physics, Romania; the Joint Institute for Nuclear Research (JINR), the Ministry of Education and Science of the Russian Federation, the National Research Centre Kurchatov Institute, the Russian Science Foundation and Russian Foundation for Basic Research, Russia; the Ministry of Education, Science, Research and Sport of the Slovak Republic, Slovakia; the National Research Foundation of South Africa, South Africa; the Swedish Research Council (VR) and the Knut \& Alice Wallenberg Foundation (KAW), Sweden; the European Organization for Nuclear Research, Switzerland; the National Science and Technology Development Agency (NSDTA), Suranaree University of Technology (SUT), and Office of the Higher Education Commission under NRU project of Thailand, Thailand; the Turkish Atomic Energy Agency (TAEK), Turkey; the National Academy of Sciences of Ukraine, Ukraine; the Science and Technology Facilities Council (STFC), United Kingdom; and the National Science Foundation of the United States of America (NSF) and the United States Department of Energy, Office of Nuclear Physics (DOE NP), United States of America.
[1] W. E. Humphrey and R. R. Ross, Low-energy interactions of $K^{-}$mesons in hydrogen, Phys. Rev. 127, 1305 (1962).

[2] M. B. Watson, M. Ferro-Luzzi, and R. D. Tripp, Analysis of $Y_{0}^{*}(1520)$ and determination of the $\Sigma$ parity, Phys. Rev. 131, 2248 (1963).

[3] T. S. Mast, M. Alston-Garnjost, R. O. Bangerter, A. S. Barbaro-Galtieri, F. T. Solmitz, and R. D. Tripp, Elastic, charge exchange, and total $K-P$ cross-sections in the momentum range $220-\mathrm{MeV} / c$ to $470-\mathrm{MeV} / c$, Phys. Rev. D 14, 13 (1976).

[4] R. J. Nowak et al., Charged $\Sigma$ hyperon production by $K^{-}$ meson interactions at rest, Nucl. Phys. B139, 61 (1978).

[5] J. Ciborowski et al., Kaon scattering and charged sigma hyperon production in $K-P$ interactions below $300-\mathrm{MEV} / C$, J. Phys. G 8, 13 (1982).

[6] D. Hadjimichef, J. Haidenbauer, and G. Krein, Short range repulsion and isospin dependence in the KN system, Phys. Rev. C 66, 055214 (2002).

[7] G. L. Shaw and M. H. Ross, Analysis of multichannel reactions, Phys. Rev. 126, 806 (1962).

[8] R. H. Dalitz and S. F. Tuan, A Possible Resonant State in Pion-Hyperon Scattering, Phys. Rev. Lett. 2, 425 (1959).

[9] R. H. Dalitz and S. F. Tuan, The phenomenological description of $K$-nucleon reaction processes, Ann. Phys. (Leipzig) 10, 307 (1960).

[10] J. M. M. Hall, W. Kamleh, D. B. Leinweber, B. J. Menadue, B. J. Owen, A. W. Thomas, and R. D. Young, Lattice QCD Evidence that the $\Lambda(1405)$ Resonance is an AntikaonNucleon Molecule, Phys. Rev. Lett. 114, 132002 (2015).

[11] N. Kaiser, P. B. Siegel, and W. Weise, Chiral dynamics and the low-energy kaon-nucleon interaction, Nucl. Phys. A594, 325 (1995).

[12] E. Oset and A. Ramos, Nonperturbative chiral approach to s-wave anti- $K N$ interactions, Nucl. Phys. A635, 99 (1998).

[13] J. A. Oller and U.G. Meißner, Chiral dynamics in the presence of bound states: Kaon nucleon interactions revisited, Phys. Lett. B 500, 263 (2001).

[14] M. F. M. Lutz and E. E. Kolomeitsev, Relativistic chiral SU(3) symmetry, large $N(c)$ sum rules and meson baryon scattering, Nucl. Phys. A700, 193 (2002).

[15] T. Hyodo and W. Weise, Effective anti- $K N$ interaction based on chiral SU(3) dynamics, Phys. Rev. C 77, 035204 (2008).

[16] Y. Kamiya, K. Miyahara, S. Ohnishi, Y. Ikeda, T. Hyodo, E. Oset, and W. Weise, Antikaon-nucleon interaction and $\Lambda(1405)$ in chiral SU(3) dynamics, Nucl. Phys. A954, 41 (2016).

[17] J. Révai, Are the chiral based $\overline{\mathrm{K}} N$ potentials really energy dependent?, Few-Body Syst. 59, 49 (2018).

[18] M. Mai and U.-G. Meißner, Constraints on the chiral unitary $\overline{\mathrm{K}} N$ from $\pi \Sigma \mathrm{K}^{+}$photoproduction data, Eur. Phys. J. A 51, 30 (2015).

[19] B. Borasoy, U. G. Meißner, and R. Nissler, $K^{-} p$ scattering length from scattering experiments, Phys. Rev. C 74, 055201 (2006).

[20] A. Cieplý and V. Krejčiř́ík, Effective model for in-medium $\bar{K} N$ interactions including the $L=1$ partial wave, Nucl. Phys. A940, 311 (2015).

[21] R. J. Hemingway, Production of $\Lambda(1405)$ in $K^{-} p$ reactions at $4.2 \mathrm{GeV} / c$, Nucl. Phys. B253, 742 (1985). 
[22] O. Braun et al., New information about the Kaon-nucleonhyperon coupling constants $\mathrm{g}(\mathrm{KN} \Sigma(1197)), \mathrm{g}(\mathrm{KN} \Sigma(1385))$ and $\mathrm{g}(\mathrm{KN} \Lambda(1405))$, Nucl. Phys. B129, 1 (1977).

[23] D. W. Thomas, A. Engler, H. E. Fisk, and R. W. Kraemer, Strange particle production from $\pi^{-} p$ interactions at $1.69 \mathrm{GeV} / c$, Nucl. Phys. B56, 15 (1973).

[24] G. Agakishiev et al. (HADES Collaboration), Baryonic resonances close to the $\overline{\mathrm{K}} N$ threshold: The case of $\Lambda(1405)$ in pp collisions, Phys. Rev. C 87, 025201 (2013).

[25] K. Moriya et al. (CLAS Collaboration), Measurement of the $\Sigma \pi$ photoproduction line shapes near the $\Lambda(1405)$, Phys. Rev. C 87, 035206 (2013).

[26] Y. Ikeda, T. Hyodo, and W. Weise, Chiral SU(3) theory of antikaon-nucleon interactions with improved threshold constraints, Nucl. Phys. A881, 98 (2012).

[27] M. Sakitt, T. B. Day, R. G. Glasser, N. Seeman, J. H. Friedman, W. E. Humphrey, and R. R. Ross, Low-energy $K^{-}$meson interactions in hydrogen, Phys. Rev. 139, B719 (1965).

[28] M. Bazzi et al. (SIDDHARTA Collaboration), A new measurement of kaonic hydrogen x-rays, Phys. Lett. B 704, 113 (2011).

[29] R. Lednicky, Correlation femtoscopy, Nucl. Phys. A774, 189 (2006).

[30] M. A. Lisa, S. Pratt, R. Soltz, and U. Wiedemann, Femtoscopy in relativistic heavy ion collisions, Annu. Rev. Nucl. Part. Sci. 55, 357 (2005).

[31] A. Kisiel, H. Zbroszczyk, and M. Szymaski, Extracting baryon-antibaryon strong interaction potentials from $p \bar{\Lambda}$ femtoscopic correlation functions, Phys. Rev. C 89, 054916 (2014).

[32] L. Adamczyk et al. (STAR Collaboration), Measurement of interaction between antiprotons, Nature (London) 527, 345 (2015).

[33] S. Acharya et al. (ALICE Collaboration), p-p, $\mathrm{p}-\Lambda$ and $\Lambda-\Lambda$ correlations studied via femtoscopy in $\mathrm{pp}$ reactions at $\sqrt{s}=7$ TeV, Phys. Rev. C 99, 024001 (2019).

[34] J. Haidenbauer, Coupled-channel effects in hadronhadron correlation functions, Nucl. Phys. A981, 1 (2019).

[35] B. Abelev et al. (ALICE Collaboration), Performance of the ALICE experiment at the CERN LHC, Int. J. Mod. Phys. A 29, 1430044 (2014).

[36] S. Acharya et al. (ALICE Collaboration), Supplemental figures: Scattering studies with low-energy kaon-proton femtoscopy in proton-proton collisions at the LHC, https://cds.cern.ch/record/2703333.

[37] E. Abbas et al. (ALICE Collaboration), Performance of the ALICE VZERO system, J. Instrum. 8, P10016 (2013).

[38] K. Aamodt et al. (ALICE Collaboration), Alignment of the ALICE inner tracking system with cosmic-ray tracks, J. Instrum. 5, P03003 (2010).

[39] J. Alme, Y. Andres, H. Appelshäuser, S. Bablok, N. Bialas et al., The ALICE TPC, a large 3-dimensional tracking device with fast readout for ultra-high multiplicity events, Nucl. Instrum. Methods Phys. Res., Sect. A 622, 316 (2010).

[40] A. Akindinov et al., Performance of the ALICE time-offlight detector at the LHC, Eur. Phys. J. Plus 128, 44 (2013).

[41] S. Acharya et al. (ALICE Collaboration), Study of the $\Lambda-\Lambda$ interaction with femtoscopy correlations in $\mathrm{pp}$ and $\mathrm{p}-\mathrm{Pb}$ collisions at the LHC, Phys. Lett. B 797, 134822 (2019).

[42] S. Acharya et al. (ALICE Collaboration), Event-shape and multiplicity dependence of freeze-out radii in pp collisions at $\sqrt{s}=7 \mathrm{TeV}$, J. High Energy Phys. 09 (2019) 108.

[43] B. Abelev et al. (ALICE Collaboration), Transverse sphericity of primary charged particles in minimum bias protonproton collisions at $\sqrt{s}=0.9,2.76$ and $7 \mathrm{TeV}$, Eur. Phys. J. C 72, 2124 (2012).

[44] M. Tanabashi et al. (Particle Data Group), Review of particle physics, Phys. Rev. D 98, 030001 (2018).

[45] T. Sjostrand, S. Mrenna, and P.Z. Skands, PYTHIA 6.4 physics and manual, J. High Energy Phys. 05 (2006) 026.

[46] T. Sjostrand, S. Mrenna, and P.Z. Skands, A brief introduction to PYTHIA 8.1, Comput. Phys. Commun. 178, 852 (2008).

[47] D. L. Mihaylov, V. Mantovani Sarti, O. W. Arnold, L. Fabbietti, B. Hohlweger, and A. M. Mathis, A femtoscopic correlation analysis tool using the Schrödinger equation (CATS), Eur. Phys. J. C 78, 394 (2018).

[48] R. Brun, F. Carminati, and S. Giani, GEANT detector description and simulation tool, Program Library Long Write-up W5013 (1994).

[49] S. Agostinelli et al. (GEANT4 Collaboration), GEANT4: A simulation toolkit, Nucl. Instrum. Methods Phys. Res., Sect. A 506, 250 (2003).

[50] J. Haidenbauer, G. Krein, U.-G. Meißner, and L. Tolos, DN interaction from meson exchange, Eur. Phys. J. A 47, 18 (2011).

[51] J. Adam et al. (ALICE Collaboration), One-dimensional pion, kaon, and proton femtoscopy in $\mathrm{Pb}-\mathrm{Pb}$ collisions at $\sqrt{s_{\mathrm{NN}}}=2.76 \mathrm{TeV}$, Phys. Rev. C 92, 054908 (2015).

[52] K. Miyahara and T. Hyodo, Structure of $\Lambda(1405)$ and construction of $\overline{\mathrm{K}} N$ local potential based on chiral SU(3) dynamics, Phys. Rev. C 93, 015201 (2016).

[53] S. Ohnishi, W. Horiuchi, T. Hoshino, K. Miyahara, and T. Hyodo, Few-body approach to structure of $\bar{K}$-nuclear quasibound states, Phys. Rev. C 95, 065202 (2017).

[54] S. Cho et al. (ExHIC Collaboration), Exotic hadrons from heavy ion collisions, Prog. Part. Nucl. Phys. 95, 279 (2017).

[55] Y. Ikeda, T. Hyodo, and W. Weise, Improved constraints on chiral SU(3) dynamics from kaonic hydrogen, Phys. Lett. B 706, 63 (2011).

[56] S. Acharya et al. (ALICE Collaboration), Measuring $\mathrm{K}_{\mathrm{S}}^{0} \mathrm{~K}^{ \pm}$ interactions using pp collisions at $\sqrt{s}=7 \mathrm{TeV}$, Phys. Lett. B 790, 22 (2019). 
S. Acharya, ${ }^{141}$ D. Adamová, ${ }^{93}$ S. P. Adhya, ${ }^{141}$ A. Adler, ${ }^{74}$ J. Adolfsson, ${ }^{80}$ M. M. Aggarwal, ${ }^{98}$ G. Aglieri Rinella, ${ }^{34}$ M. Agnello, ${ }^{31}$ N. Agrawal, ${ }^{10}$ Z. Ahammed, ${ }^{141}$ S. Ahmad, ${ }^{17}$ S. U. Ahn, ${ }^{76}$ S. Aiola, ${ }^{146}$ A. Akindinov, ${ }^{64}$ M. Al-Turany, ${ }^{105}$ S. N. Alam, ${ }^{141}$ D. S. D. Albuquerque, ${ }^{122}$ D. Aleksandrov, ${ }^{87}$ B. Alessandro,${ }^{58}$ H. M. Alfanda, ${ }^{6}$ R. Alfaro Molina, ${ }^{72}$ B. Ali, ${ }^{17}$ Y. Ali, ${ }^{15}$ A. Alici, ${ }^{10,53,27 a, 27 b}$ A. Alkin, ${ }^{2}$ J. Alme, ${ }^{22}$ T. Alt, ${ }^{69}$ L. Altenkamper, ${ }^{22}$ I. Altsybeev, ${ }^{112}$ M. N. Anaam, ${ }^{6}$ C. Andrei, ${ }^{47}$ D. Andreou, ${ }^{34}$ H. A. Andrews, ${ }^{109}$ A. Andronic, ${ }^{144}$ M. Angeletti, ${ }^{34}$ V. Anguelov, ${ }^{102}$ C. Anson, ${ }^{16}$ T. Antičić, ${ }^{106}$ F. Antinori,${ }^{56}$ P. Antonioli, ${ }^{53}$ R. Anwar, ${ }^{126}$ N. Apadula,${ }^{79}$ L. Aphecetche, ${ }^{114}$ H. Appelshäuser, ${ }^{69}$ S. Arcelli, ${ }^{27 a, 27 b}$ R. Arnaldi,${ }^{58}$ M. Arratia, ${ }^{79}$ I. C. Arsene, ${ }^{21}$ M. Arslandok, ${ }^{102}$ A. Augustinus,${ }^{34}$ R. Averbeck, ${ }^{105}$ S. Aziz, ${ }^{61}$ M. D. Azmi, ${ }^{17}$ A. Badalà, ${ }^{55}$ Y. W. Baek, ${ }^{40}$ S. Bagnasco, ${ }^{58}$ R. Bailhache, ${ }^{69}$ R. Bala ${ }^{99}$ A. Baldisseri, ${ }^{137}$ M. Ball, ${ }^{42}$ R. C. Baral,${ }^{85}$ R. Barbera, ${ }^{28 a, 28 b}$ L. Barioglio, ${ }^{26 a, 26 b}$ G. G. Barnaföldi, ${ }^{145}$ L. S. Barnby, ${ }^{92}$ V. Barret, ${ }^{134}$ P. Bartalini, ${ }^{6}$ K. Barth,${ }^{34}$ E. Bartsch, ${ }^{69}$ F. Baruffaldi, ${ }^{29 a, 29 b}$ N. Bastid, ${ }^{134}$ S. Basu, ${ }^{143}$ G. Batigne, ${ }^{114}$ B. Batyunya ${ }^{75}$ P. C. Batzing, ${ }^{21}$ D. Bauri, ${ }^{48}$ J. L. Bazo Alba ${ }^{110}$ I. G. Bearden,${ }^{88}$ C. Bedda,${ }^{63}$ N. K. Behera ${ }^{60}$ I. Belikov, ${ }^{136}$ F. Bellini,${ }^{34}$ R. Bellwied, ${ }^{126}$ V. Belyaev,${ }^{91}$ G. Bencedi, ${ }^{145}$ S. Beole, ${ }^{26 a, 26 b}$ A. Bercuci, ${ }^{47}$ Y. Berdnikov, ${ }^{96}$ D. Berenyi, ${ }^{145}$ R. A. Bertens, ${ }^{130}$ D. Berzano,${ }^{58}$ L. Betev,${ }^{34}$ A. Bhasin, ${ }^{99}$ I. R. Bhat,${ }^{99}$ H. Bhatt, ${ }^{48}$ B. Bhattacharjee, ${ }^{41}$ A. Bianchi, ${ }^{26 a, 26 b}$ L. Bianchi, ${ }^{126,26 a, 26 b}$ N. Bianchi, ${ }^{51}$ J. Bielčík, ${ }^{37}$ J. Bielčíková, ${ }^{93}$ A. Bilandzic, ${ }^{103,117}$ G. Biro, ${ }^{145}$ R. Biswas, ${ }^{3 a, 3 b}$ S. Biswas, ${ }^{3 a, 3 b}$ J. T. Blair, ${ }^{119}$ D. Blau, ${ }^{87}$ C. Blume, ${ }^{69}$ G. Boca, ${ }^{139}$ F. Bock,${ }^{34,94}$ A. Bogdanov, ${ }^{91}$ L. Boldizsár, ${ }^{145}$ A. Bolozdynya, ${ }^{91}$ M. Bombara,${ }^{38}$ G. Bonomi, ${ }^{140}$ M. Bonora, ${ }^{34}$ H. Borel, ${ }^{137}$ A. Borissov, ${ }^{144,91}$ M. Borri, ${ }^{128}$ H. Bossi, ${ }^{146}$ E. Botta, ${ }^{26 a, 26 b}$ C. Bourjau, ${ }^{88}$ L. Bratrud, ${ }^{69}$ P. Braun-Munzinger, ${ }^{105}$ M. Bregant, ${ }^{121}$ T. A. Broker, ${ }^{69}$ M. Broz,${ }^{37}$ E. J. Brucken, ${ }^{43}$ E. Bruna ${ }^{58}$ G. E. Bruno, ${ }^{33 a, 33 b, 104}$ M. D. Buckland, ${ }^{128}$ D. Budnikov, ${ }^{107}$ H. Buesching, ${ }^{69}$ S. Bufalino, ${ }^{31}$ O. Bugnon, ${ }^{114}$ P. Buhler, ${ }^{113}$ P. Buncic, ${ }^{34}$ O. Busch, ${ }^{133, a}$ Z. Buthelezi, ${ }^{73}$ J. B. Butt, ${ }^{15}$ J. T. Buxton, ${ }^{95}$ D. Caffarri, ${ }^{89}$ A. Caliva, ${ }^{105}$

E. Calvo Villar, ${ }^{110}$ R. S. Camacho, ${ }^{44}$ P. Camerini, ${ }^{25 a, 25 b}$ A. A. Capon, ${ }^{113}$ F. Carnesecchi, ${ }^{10}$ J. Castillo Castellanos, ${ }^{137}$

A. J. Castro, ${ }^{130}$ E. A. R. Casula, ${ }^{54}$ F. Catalano, ${ }^{31}$ C. Ceballos Sanchez, ${ }^{52}$ P. Chakraborty, ${ }^{48}$ S. Chandra, ${ }^{141}$ B. Chang, ${ }^{127}$ W. Chang, ${ }^{6}$ S. Chapeland, ${ }^{34}$ M. Chartier, ${ }^{128}$ S. Chattopadhyay, ${ }^{141}$ S. Chattopadhyay, ${ }^{108}$ A. Chauvin, ${ }^{24 a, 24 b}$ C. Cheshkov, ${ }^{135}$ B. Cheynis, ${ }^{135}$ V. Chibante Barroso, ${ }^{34}$ D. D. Chinellato, ${ }^{122}$ S. Cho,${ }^{60}$ P. Chochula, ${ }^{34}$ T. Chowdhury, ${ }^{134}$ P. Christakoglou, ${ }^{89}$ C. H. Christensen ${ }^{88}$ P. Christiansen, ${ }^{80}$ T. Chujo, ${ }^{133}$ C. Cicalo, ${ }^{54}$ L. Cifarelli, ${ }^{10,27 a, 27 b}$ F. Cindolo, ${ }^{53}$ J. Cleymans, ${ }^{125}$ F. Colamaria, ${ }^{52}$ D. Colella, ${ }^{52}$ A. Collu, ${ }^{79}$ M. Colocci, ${ }^{27,27 b}$ M. Concas,${ }^{58, b}$ G. Conesa Balbastre, ${ }^{78}$ Z. Conesa del Valle, ${ }^{61}$ G. Contin, ${ }^{128}$ J. G. Contreras, ${ }^{37}$ T. M. Cormier, ${ }^{94}$ Y. Corrales Morales, ${ }^{26 a, 26 b, 58}$ P. Cortese, ${ }^{32}$ M. R. Cosentino, ${ }^{123}$ F. Costa, ${ }^{34}$ S. Costanza, ${ }^{139}$ J. Crkovská, ${ }^{61}$ P. Crochet, ${ }^{134}$ E. Cuautle ${ }^{70}$ L. Cunqueiro, ${ }^{94}$ D. Dabrowski, ${ }^{142}$ T. Dahms, ${ }^{103,117}$ A. Dainese,${ }^{56}$ F. P. A. Damas, ${ }^{137,114}$ S. Dani, ${ }^{66}$ M. C. Danisch ${ }^{102}$ A. Danu, ${ }^{68}$ D. Das, ${ }^{108}$ I. Das, ${ }^{108}$ S. Das, ${ }^{3 a, 3 b}$ A. Dash, ${ }^{85}$ S. Dash,${ }^{48}$ A. Dashi, ${ }^{103}$ S. De ${ }^{85,49}$ A. De Caro, ${ }^{30 a, 30 b}$ G. de Cataldo,${ }^{52}$ C. de Conti, ${ }^{121}$ J. de Cuveland, ${ }^{39}$ A. De Falco, ${ }^{24 a, 24 b}$ D. De Gruttola, ${ }^{10}$ N. De Marco,${ }^{58}$ S. De Pasquale, ${ }^{30 a, 30 b}$ R. D. De Souza, ${ }^{122}$ S. Deb,${ }^{49}$ H. F. Degenhardt, ${ }^{121}$ A. Deisting, ${ }^{102,105}$ K. R. Deja, ${ }^{142}$ A. Deloff, ${ }^{84}$ S. Delsanto, ${ }^{131,26 a, 26 b}$ P. Dhankher, ${ }^{48}$ D. Di Bari,${ }^{33 a, 33 b}$ A. Di Mauro, ${ }^{34}$ R. A. Diaz,${ }^{8}$ T. Dietel, ${ }^{125}$ P. Dillenseger, ${ }^{69}$ Y. Ding, ${ }^{6}$ R. Divià, ${ }^{34} \varnothing$. Djuvsland, ${ }^{22}$ U. Dmitrieva, ${ }^{62}$ A. Dobrin,${ }^{34,68}$ B. Dönigus, ${ }^{69}$ O. Dordic, ${ }^{21}$

A. K. Dubey, ${ }^{141}$ A. Dubla, ${ }^{105}$ S. Dudi, ${ }^{98}$ A. K. Duggal, ${ }^{98}$ M. Dukhishyam, ${ }^{85}$ P. Dupieux, ${ }^{134}$ R. J. Ehlers, ${ }^{146}$ D. Elia, ${ }^{52}$ H. Engel,${ }^{74}$ E. Epple, ${ }^{146}$ B. Erazmus, ${ }^{114}$ F. Erhardt, ${ }^{97}$ A. Erokhin, ${ }^{112}$ M. R. Ersdal, ${ }^{22}$ B. Espagnon, ${ }^{61}$ G. Eulisse,${ }^{34}$ J. Eum, ${ }^{18}$ D. Evans, ${ }^{109}$ S. Evdokimov, ${ }^{90}$ L. Fabbietti, ${ }^{17,103}$ M. Faggin, ${ }^{29 a, 29 b}$ J. Faivre, ${ }^{78}$ A. Fantoni, ${ }^{51}$ M. Fasel, ${ }^{94}$ P. Fecchio, ${ }^{31}$

L. Feldkamp, ${ }^{144}$ A. Feliciello, ${ }^{58}$ G. Feofilov, ${ }^{112}$ A. Fernández Téllez, ${ }^{44}$ A. Ferrero, ${ }^{137}$ A. Ferretti, ${ }^{26 a, 26 b}$ A. Festanti, ${ }^{34}$ V. J. G. Feuillard, ${ }^{102}$ J. Figiel, ${ }^{118}$ S. Filchagin, ${ }^{107}$ D. Finogeev, ${ }^{62}$ F. M. Fionda, ${ }^{22}$ G. Fiorenza, ${ }^{52}$ F. Flor, ${ }^{126}$ S. Foertsch, ${ }^{73}$ P. Foka ${ }^{105}$ S. Fokin, ${ }^{87}$ E. Fragiacomo, ${ }^{59}$ A. Francisco, ${ }^{114}$ U. Frankenfeld, ${ }^{105}$ G. G. Fronze, ${ }^{26 a, 26 b}$ U. Fuchs,${ }^{34}$ C. Furget ${ }^{78}$

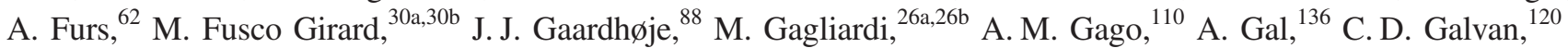
P. Ganoti, ${ }^{83}$ C. Garabatos, ${ }^{105}$ E. Garcia-Solis, ${ }^{11}$ K. Garg, ${ }^{28 a, 28 b}$ C. Gargiulo, ${ }^{34}$ K. Garner, ${ }^{144}$ P. Gasik, ${ }^{103,117}$ E. F. Gauger, ${ }^{119}$ M. B. Gay Ducati, ${ }^{71}$ M. Germain, ${ }^{114}$ J. Ghosh, ${ }^{108}$ P. Ghosh, ${ }^{141}$ S. K. Ghosh, ${ }^{3 a, 3 b}$ P. Gianotti, ${ }^{51}$ P. Giubellino, ${ }^{105,58}$ P. Giubilato, ${ }^{29 a, 29 b}$ P. Glässel, ${ }^{102}$ D. M. Goméz Coral, ${ }^{72}$ A. Gomez Ramirez, ${ }^{74}$ V. Gonzalez, ${ }^{105}$ P. González-Zamora, ${ }^{44}$ S. Gorbunov, ${ }^{39}$ L. Görlich, ${ }^{118}$ S. Gotovac, ${ }^{35}$ V. Grabski, ${ }^{72}$ L. K. Graczykowski, ${ }^{142}$ K. L. Graham, ${ }^{109}$ L. Greiner, ${ }^{79}$ A. Grelli, ${ }^{63}$ C. Grigoras ${ }^{34}$ V. Grigoriev ${ }^{91}$ A. Grigoryan, ${ }^{1}$ S. Grigoryan, ${ }^{75}$ O. S. Groettvik, ${ }^{22}$ J. M. Gronefeld, ${ }^{105}$ F. Grosa, ${ }^{31}$ J. F. Grosse-Oetringhaus, ${ }^{34}$ R. Grosso, ${ }^{105}$ R. Guernane,${ }^{78}$ B. Guerzoni, ${ }^{27 a, 27 b}$ M. Guittiere, ${ }^{114}$ K. Gulbrandsen,${ }^{88}$ T. Gunji, ${ }^{132}$ A. Gupta, ${ }^{99}$ R. Gupta, ${ }^{99}$ I. B. Guzman, ${ }^{44}$ R. Haake, ${ }^{146,34}$ M. K. Habib ${ }^{105}$ C. Hadjidakis, ${ }^{61}$ H. Hamagaki, ${ }^{81}$ G. Hamar, ${ }^{145}$ M. Hamid, ${ }^{6}$ J. C. Hamon, ${ }^{136}$ R. Hannigan, ${ }^{119}$ M. R. Haque, ${ }^{63}$ A. Harlenderova, ${ }^{105}$ J. W. Harris, ${ }^{146}$ A. Harton, ${ }^{11}$ H. Hassan, ${ }^{78}$ D. Hatzifotiadou, ${ }^{10,53}$ P. Hauer, ${ }^{42}$ S. Hayashi, ${ }^{132}$ S. T. Heckel,${ }^{69}$ E. Hellbär, ${ }^{69}$ H. Helstrup, ${ }^{36}$ A. Herghelegiu, ${ }^{47}$

E. G. Hernandez, ${ }^{44}$ G. Herrera Corral, ${ }^{9}$ F. Herrmann, ${ }^{144}$ K. F. Hetland,${ }^{36}$ T. E. Hilden ${ }^{43}$ H. Hillemanns,${ }^{34}$ C. Hills, ${ }^{128}$ B. Hippolyte, ${ }^{136}$ B. Hohlweger, ${ }^{103}$ D. Horak, ${ }^{37}$ S. Hornung, ${ }^{105}$ R. Hosokawa, ${ }^{133}$ P. Hristov, ${ }^{34}$ C. Huang, ${ }^{61}$ C. Hughes, ${ }^{130}$ 
P. Huhn, ${ }^{69}$ T. J. Humanic, ${ }^{95}$ H. Hushnud, ${ }^{108}$ L. A. Husova, ${ }^{144}$ N. Hussain, ${ }^{41}$ S. A. Hussain, ${ }^{15}$ T. Hussain, ${ }^{17}$ D. Hutter, ${ }^{39}$ D. S. Hwang, ${ }^{19}$ J. P. Iddon, ${ }^{128}$ R. Ilkaev ${ }^{107}$ M. Inaba, ${ }^{133}$ M. Ippolitov, ${ }^{87}$ M. S. Islam, ${ }^{108}$ M. Ivanov, ${ }^{105}$ V. Ivanov, ${ }^{96}$ V. Izucheev, ${ }^{90}$ B. Jacak, ${ }^{79}$ N. Jacazio, ${ }^{27 a, 27 b}$ P. M. Jacobs, ${ }^{79}$ M. B. Jadhav, ${ }^{48}$ S. Jadlovska, ${ }^{116}$ J. Jadlovsky, ${ }^{116}$ S. Jaelani ${ }^{63}$ C. Jahnke, ${ }^{121}$ M. J. Jakubowska, ${ }^{142}$ M. A. Janik, ${ }^{142}$ M. Jercic, ${ }^{97}$ O. Jevons, ${ }^{109}$ R. T. Jimenez Bustamante, ${ }^{105}$ M. Jin, ${ }^{126}$ F. Jonas, ${ }^{144,94}$ P. G. Jones, ${ }^{109}$ A. Jusko, ${ }^{109}$ P. Kalinak, ${ }^{65}$ A. Kalweit,${ }^{34}$ J. H. Kang, ${ }^{147}$ V. Kaplin, ${ }^{91}$ S. Kar, ${ }^{6}$ A. Karasu Uysal, ${ }^{77}$ O. Karavichev, ${ }^{62}$ T. Karavicheva, ${ }^{62}$ P. Karczmarczyk, ${ }^{34}$ E. Karpechev, ${ }^{62}$ U. Kebschull, ${ }^{74}$ R. Keidel,${ }^{46}$ M. Keil, ${ }^{34}$ B. Ketzer ${ }^{42}$ Z. Khabanova, ${ }^{89}$ A. M. Khan, ${ }^{6}$ S. Khan, ${ }^{17}$ S. A. Khan, ${ }^{141}$ A. Khanzadeev, ${ }^{96}$ Y. Kharlov, ${ }^{90}$ A. Khatun, ${ }^{17}$ A. Khuntia, ${ }^{118,49}$ B. Kileng, ${ }^{36}$ B. Kim, ${ }^{60}$ B. Kim, ${ }^{133}$ D. Kim, ${ }^{147}$ D. J. Kim, ${ }^{127}$ E. J. Kim, ${ }^{13}$ H. Kim, ${ }^{147}$ J. S. Kim, ${ }^{40}$ J. Kim, ${ }^{102}$ J. Kim, ${ }^{147}$ J. Kim, ${ }^{13}$ M. Kim, ${ }^{102}$ S. Kim, ${ }^{19}$ T. Kim, ${ }^{147}$ T. Kim, ${ }^{147}$ K. Kindra, ${ }^{98}$ S. Kirsch,${ }^{39}$ I. Kisel,,${ }^{39}$ S. Kiselev, ${ }^{64}$ A. Kisiel,,${ }^{142}$ J. L. Klay, ${ }^{5}$ C. Klein ${ }^{69}$ J. Klein, ${ }^{58}$ S. Klein, ${ }^{79}$ C. Klein-Bösing, ${ }^{144}$ S. Klewin, ${ }^{102}$ A. Kluge,${ }^{34}$ M. L. Knichel, ${ }^{34}$ A. G. Knospe, ${ }^{126}$ C. Kobdaj, ${ }^{115}$ M. K. Köhler, ${ }^{102}$ T. Kollegger,${ }^{105}$ A. Kondratyev, ${ }^{75}$ N. Kondratyeva, ${ }^{91}$ E. Kondratyuk, ${ }^{90}$ P. J. Konopka, ${ }^{34}$ L. Koska, ${ }^{116}$ O. Kovalenko, ${ }^{84}$ V. Kovalenko, ${ }^{112}$ M. Kowalski, ${ }^{118}$ I. Králik, ${ }^{65}$ A. Kravčáková, ${ }^{38}$ L. Kreis, ${ }^{105}$ M. Krivda, ${ }^{65,109}$ F. Krizek, ${ }^{93}$ K. Krizkova Gajdosova,${ }^{37}$ M. Krüger, ${ }^{69}$ E. Kryshen,${ }^{96}$ M. Krzewicki, ${ }^{39}$ A. M. Kubera, ${ }^{95}$ V. Kučera, ${ }^{60}$ C. Kuhn, ${ }^{136}$ P. G. Kuijer, ${ }^{89}$ L. Kumar, ${ }^{98}$ S. Kumar ${ }^{48}$ S. Kundu, ${ }^{85}$ P. Kurashvili,${ }^{84}$ A. Kurepin, ${ }^{62}$ A. B. Kurepin, ${ }^{62}$ S. Kushpil ${ }^{93}$

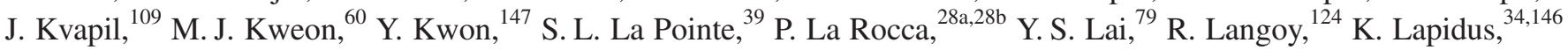
A. Lardeux,${ }^{21}$ P. Larionov, ${ }^{51}$ E. Laudi, ${ }^{34}$ R. Lavicka, ${ }^{37}$ T. Lazareva, ${ }^{112}$ R. Lea,${ }^{25 a, 25 b}$ L. Leardini, ${ }^{102}$ S. Lee, ${ }^{147}$ F. Lehas, ${ }^{89}$ S. Lehner, ${ }^{113}$ J. Lehrbach, ${ }^{39}$ R. C. Lemmon, ${ }^{92}$ I. León Monzón, ${ }^{120}$ E. D. Lesser, ${ }^{20}$ M. Lettrich, ${ }^{34}$ P. Lévai, ${ }^{145}$ X. Li,${ }^{12}$ X. L. Li, ${ }^{6}$ J. Lien, ${ }^{124}$ R. Lietava, ${ }^{109}$ B. Lim, ${ }^{18}$ S. Lindal, ${ }^{21}$ V. Lindenstruth, ${ }^{39}$ S. W. Lindsay, ${ }^{128}$ C. Lippmann, ${ }^{105}$ M. A. Lisa, ${ }^{95}$ V. Litichevskyi, ${ }^{43}$ A. Liu, ${ }^{79}$ S. Liu, ${ }^{95}$ H. M. Ljunggren, ${ }^{80}$ W. J. Llope,${ }^{143}$ I. M. Lofnes, ${ }^{22}$ V. Loginov, ${ }^{91}$ C. Loizides, ${ }^{94}$ P. Loncar, ${ }^{35}$ X. Lopez, ${ }^{134}$ E. López Torres, ${ }^{8}$ P. Luettig, ${ }^{69}$ J. R. Luhder ${ }^{144}$ M. Lunardon, ${ }^{29 a, 29 b}$ G. Luparello, ${ }^{59}$ M. Lupi ${ }^{34}$ A. Maevskaya, ${ }^{62}$ M. Mager, ${ }^{34}$ S. M. Mahmood, ${ }^{21}$ T. Mahmoud, ${ }^{42}$ A. Maire, ${ }^{136}$ R. D. Majka, ${ }^{146}$ M. Malaev, ${ }^{96}$ Q. W. Malik, ${ }^{21}$ L. Malinina, ${ }^{75, \mathrm{c}}$ D. Mal'Kevich, ${ }^{64}$ P. Malzacher, ${ }^{105}$ A. Mamonov, ${ }^{107}$ V. Manko, ${ }^{87}$ F. Manso, ${ }^{134}$ V. Manzari, ${ }^{52}$ Y. Mao, ${ }^{6}$ M. Marchisone, ${ }^{135}$ J. Mareš,${ }^{67}$ G. V. Margagliotti, ${ }^{25 a, 25 b}$ A. Margotti, ${ }^{53}$ J. Margutti, ${ }^{63}$ A. Marín, ${ }^{105}$ C. Markert, ${ }^{119}$ M. Marquard, ${ }^{69}$ N. A. Martin, ${ }^{102}$ P. Martinengo, ${ }^{34}$ J. L. Martinez, ${ }^{126}$ M. I. Martínez, ${ }^{44}$ G. Martínez García, ${ }^{114}$ M. Martinez Pedreira, ${ }^{34}$ S. Masciocchi, ${ }^{105}$ M. Masera, ${ }^{26 a, 26 b}$ A. Masoni, ${ }^{54}$ L. Massacrier, ${ }^{61}$ E. Masson, ${ }^{114}$ A. Mastroserio, ${ }^{52,138}$ A. M. Mathis, ${ }^{103,117}$ P. F. T. Matuoka, ${ }^{121}$ A. Matyja, ${ }^{118}$ C. Mayer, ${ }^{118}$ M. Mazzilli, $, 33,33 b$ M. A. Mazzoni ${ }^{57}$ A. F. Mechler ${ }^{69}$ F. Meddi, ${ }^{23 a, 23 b}$ Y. Melikyan, ${ }^{91}$ A. Menchaca-Rocha, ${ }^{72}$ E. Meninno, ${ }^{30 a, 30 b}$ M. Meres,${ }^{14}$ S. Mhlanga, ${ }^{125}$ Y. Miake, ${ }^{133}$ L. Micheletti, ${ }^{26 a, 26 b}$ M. M. Mieskolainen, ${ }^{43}$ D. L. Mihaylov, ${ }^{103}$ K. Mikhaylov, ${ }^{64,75}$ A. Mischke, ${ }^{63, a}$ A. N. Mishra, ${ }^{70}$ D. Miśkowiec, ${ }^{105}$ C. M. Mitu, ${ }^{68}$ N. Mohammadi, ${ }^{34}$ A. P. Mohanty, ${ }^{63}$ B. Mohanty, ${ }^{85}$ M. Mohisin Khan,${ }^{17, d}$ M. Mondal, ${ }^{141}$ M. M. Mondal, ${ }^{66}$ C. Mordasini, ${ }^{103}$ D. A. Moreira De Godoy ${ }^{144}$ L. A. P. Moreno, ${ }^{44}$ S. Moretto, ${ }^{29 a, 29 b}$ A. Morreale, ${ }^{114}$ A. Morsch, ${ }^{34}$ T. Mrnjavac, ${ }^{34}$ V. Muccifora, ${ }^{51}$ E. Mudnic,${ }^{35}$ D. Mühlheim, ${ }^{144}$ S. Muhuri, ${ }^{141}$ J. D. Mulligan, ${ }^{79,146}$ M. G. Munhoz, ${ }^{121}$ K. Münning, ${ }^{42}$ R. H. Munzer ${ }^{69}$ H. Murakami, ${ }^{132}$ S. Murray, ${ }^{73}$ L. Musa, ${ }^{34}$ J. Musinsky, ${ }^{65}$ C. J. Myers, ${ }^{126}$ J. W. Myrcha,${ }^{142}$ B. Naik, ${ }^{48}$ R. Nair, ${ }^{84}$ B. K. Nandi, ${ }^{48}$ R. Nania, ${ }^{10,53}$ E. Nappi,${ }^{52}$ M. U. Naru, ${ }^{15}$ A. F. Nassirpour, ${ }^{80}$ H. Natal da Luz, ${ }^{121}$ C. Nattrass, ${ }^{130}$ R. Nayak,${ }^{48}$ T. K. Nayak, ${ }^{85,141}$ S. Nazarenko, ${ }^{107}$

R. A. Negrao De Oliveira, ${ }^{69}$ L. Nellen, ${ }^{70}$ S. V. Nesbo, ${ }^{36}$ G. Neskovic, ${ }^{39}$ B. S. Nielsen,${ }^{88}$ S. Nikolaev, ${ }^{87}$ S. Nikulin, ${ }^{87}$ V. Nikulin, ${ }^{96}$ F. Noferini, ${ }^{10,53}$ P. Nomokonov, ${ }^{75}$ G. Nooren, ${ }^{63}$ J. Norman, ${ }^{78}$ P. Nowakowski, ${ }^{142}$ A. Nyanin, ${ }^{87}$ J. Nystrand, ${ }^{22}$ M. Ogino, ${ }^{81}$ A. Ohlson, ${ }^{102}$ J. Oleniacz, ${ }^{142}$ A. C. Oliveira Da Silva, ${ }^{121}$ M. H. Oliver, ${ }^{146}$ J. Onderwaater, ${ }^{105}$ C. Oppedisano, ${ }^{58}$ R. Orava ${ }^{43}$ A. Ortiz Velasquez, ${ }^{70}$ A. Oskarsson, ${ }^{80}$ J. Otwinowski, ${ }^{118}$ K. Oyama, ${ }^{81}$ Y. Pachmayer, ${ }^{102}$ V. Pacik, ${ }^{88}$ D. Pagano, ${ }^{140}$ G. Paić, ${ }^{70}$ P. Palni, ${ }^{6}$ J. Pan, ${ }^{143}$ A. K. Pandey, ${ }^{48}$ S. Panebianco, ${ }^{137}$ V. Papikyan, ${ }^{1}$ P. Pareek, ${ }^{49}$ J. Park, ${ }^{60}$ J. E. Parkkila, ${ }^{127}$ S. Parmar, ${ }^{98}$ A. Passfeld, ${ }^{144}$ S. P. Pathak,${ }^{126}$ R. N. Patra, ${ }^{141}$ B. Paul, ${ }^{58}$ H. Pei, ${ }^{6}$ T. Peitzmann, ${ }^{63}$ X. Peng, ${ }^{6}$ L. G. Pereira,${ }^{71}$ H. Pereira Da Costa, ${ }^{137}$ D. Peresunko, ${ }^{87}$ G. M. Perez, ${ }^{8}$ E. Perez Lezama, ${ }^{69}$ V. Peskov,${ }^{69}$ Y. Pestov, ${ }^{4}$ V. Petráček, ${ }^{37}$ M. Petrovici, ${ }^{47}$ R. P. Pezzi, ${ }^{71}$ S. Piano, ${ }^{59}$ M. Pikna, ${ }^{14}$ P. Pillot, ${ }^{114}$ L. O. D. L. Pimentel,${ }^{88}$ O. Pinazza, ${ }^{53,34}$ L. Pinsky, ${ }^{126}$ S. Pisano, ${ }^{51}$ D. B. Piyarathna, ${ }^{126}$ M. Płoskon,${ }^{79}$ M. Planinic, ${ }^{97}$ F. Pliquett, ${ }^{69}$ J. Pluta, ${ }^{142}$ S. Pochybova, ${ }^{145}$ M. G. Poghosyan, ${ }^{94}$ B. Polichtchouk, ${ }^{90}$ N. Poljak, ${ }^{97}$ W. Poonsawat, ${ }^{115}$ A. Pop,${ }^{47}$ H. Poppenborg, ${ }^{144}$ S. Porteboeuf-Houssais,${ }^{134}$ V. Pozdniakov, ${ }^{75}$ S. K. Prasad, ${ }^{3 a, 3 b}$ R. Preghenella, ${ }^{53}$ F. Prino, ${ }^{58}$ C. A. Pruneau, ${ }^{143}$ I. Pshenichnov, ${ }^{62}$ M. Puccio, ${ }^{26 a, 26 b, 34}$ V. Punin, ${ }^{107}$ K. Puranapanda, ${ }^{141}$ J. Putschke, ${ }^{143}$ R. E. Quishpe, ${ }^{126}$ S. Ragoni, ${ }^{109}$ S. Raha,${ }^{3 a, 3 b}$ S. Rajput, ${ }^{99}$ J. Rak, ${ }^{127}$ A. Rakotozafindrabe, ${ }^{137}$ L. Ramello, ${ }^{32}$ F. Rami, ${ }^{136}$ R. Raniwala, ${ }^{100}$ S. Raniwala, ${ }^{100}$ S. S. Räsänen, ${ }^{43}$ B. T. Rascanu, ${ }^{69}$ R. Rath ${ }^{49}$ V. Ratza ${ }^{42}$ I. Ravasenga ${ }^{31}$ K. F. Read, ${ }^{130,94}$ K. Redlich,${ }^{84, e}$ A. Rehman, ${ }^{22}$ P. Reichelt, ${ }^{69}$ F. Reidt,${ }^{34}$ X. Ren ${ }^{6}$ R. Renfordt, ${ }^{69}$ A. Reshetin, ${ }^{62}$ J.-P. Revol, ${ }^{10}$ K. Reygers, ${ }^{102}$ V. Riabov, ${ }^{96}$ T. Richert, ${ }^{80,88}$ M. Richter, ${ }^{21}$ P. Riedler, ${ }^{34}$ 
W. Riegler, ${ }^{34}$ F. Riggi, ${ }^{28 a, 28 b}$ C. Ristea ${ }^{68}$ S. P. Rode,${ }^{49}$ M. Rodríguez Cahuantzi,${ }^{44}$ K. Røed,${ }^{21}$ R. Rogalev, ${ }^{90}$ E. Rogochaya,${ }^{75}$ D. Rohr ${ }^{34}$ D. Röhrich, ${ }^{22}$ P. S. Rokita, ${ }^{142}$ F. Ronchetti, ${ }^{51}$ E. D. Rosas, ${ }^{70}$ K. Roslon, ${ }^{142}$ P. Rosnet,${ }^{134}$ A. Rossi, ${ }^{56,29 a, 29 b}$ A. Rotondi, ${ }^{139}$ F. Roukoutakis, ${ }^{83}$ A. Roy ${ }^{49}$ P. Roy,${ }^{108}$ O. V. Rueda, ${ }^{80}$ R. Rui, ${ }^{25 a, 25 b}$ B. Rumyantsev, ${ }^{75}$ A. Rustamov, ${ }^{86}$ E. Ryabinkin, ${ }^{87}$ Y. Ryabov, ${ }^{96}$ A. Rybicki, ${ }^{118}$ H. Rytkonen, ${ }^{127}$ S. Saarinen, ${ }^{43}$ S. Sadhu, ${ }^{141}$ S. Sadovsky, ${ }^{90}$ K. Šafařík,${ }^{37,34}$ S. K. Saha, ${ }^{141}$ B. Sahoo, ${ }^{48}$ P. Sahoo, ${ }^{49}$ R. Sahoo, ${ }^{49}$ S. Sahoo, ${ }^{66}$ P. K. Sahu, ${ }^{66}$ J. Saini, ${ }^{141}$ S. Sakai, ${ }^{133}$ S. Sambyal,${ }^{99}$ V. Samsonov, ${ }^{96,91}$ A. Sandoval, ${ }^{72}$ A. Sarkar ${ }^{73}$ D. Sarkar, ${ }^{141,143}$ N. Sarkar, ${ }^{141}$ P. Sarma,${ }^{41}$ V. M. Sarti, ${ }^{103}$ M. H. P. Sas, ${ }^{63}$ E. Scapparone, ${ }^{53}$ B. Schaefer, ${ }^{94}$ J. Schambach, ${ }^{119}$ H. S. Scheid ${ }^{69}$ C. Schiaua, ${ }^{47}$ R. Schicker, ${ }^{102}$ A. Schmah,${ }^{102}$ C. Schmidt, ${ }^{105}$ H. R. Schmidt, ${ }^{101}$ M. O. Schmidt, ${ }^{102}$ M. Schmidt, ${ }^{101}$ N. V. Schmidt, ${ }^{94,69}$ A. R. Schmier, ${ }^{130}$ J. Schukraft, ${ }^{34,88}$ Y. Schutz, ${ }^{34,136}$ K. Schwarz, ${ }^{105}$ K. Schweda, ${ }^{105}$ G. Scioli, ${ }^{27 a, 27 b}$ E. Scomparin, ${ }^{58}$ M. Šefč́ik, ${ }^{38}$ J. E. Seger, ${ }^{16}$ Y. Sekiguchi, ${ }^{132}$ D. Sekihata, ${ }^{45}$ I. Selyuzhenkov, ${ }^{105,91}$ S. Senyukov, ${ }^{136}$ E. Serradilla, ${ }^{72}$ P. Sett, ${ }^{48}$ A. Sevcenco, ${ }^{68}$ A. Shabanov, ${ }^{62}$ A. Shabetai, ${ }^{114}$ R. Shahoyan, ${ }^{34}$ W. Shaikh, ${ }^{108}$ A. Shangaraev, ${ }^{90}$ A. Sharma, ${ }^{98}$ A. Sharma, ${ }^{99}$ M. Sharma, ${ }^{99}$ N. Sharma, ${ }^{98}$ A. I. Sheikh, ${ }^{141}$ K. Shigaki, ${ }^{45}$ M. Shimomura, ${ }^{82}$ S. Shirinkin, ${ }^{64}$ Q. Shou, ${ }^{111}$ Y. Sibiriak,${ }^{87}$ S. Siddhanta, ${ }^{54}$ T. Siemiarczuk, ${ }^{84}$ D. Silvermyr,${ }^{80}$ G. Simatovic, ${ }^{89}$ G. Simonetti, ${ }^{103,34}$ R. Singh, ${ }^{85}$ R. Singh, ${ }^{99}$ V. K. Singh, ${ }^{141}$ V. Singhal, ${ }^{141}$ T. Sinha, ${ }^{108}$ B. Sitar, ${ }^{14}$ M. Sitta, ${ }^{32}$

T. B. Skaali, ${ }^{21}$ M. Slupecki, ${ }^{127}$ N. Smirnov, ${ }^{146}$ R. J. M. Snellings, ${ }^{63}$ T. W. Snellman, ${ }^{127}$ J. Sochan, ${ }^{116}$ C. Soncco, ${ }^{110}$ J. Song, ${ }^{60,126}$ A. Songmoolnak, ${ }^{115}$ F. Soramel, ${ }^{29 a, 29 b}$ S. Sorensen, ${ }^{130}$ I. Sputowska, ${ }^{118}$ J. Stachel, ${ }^{102}$ I. Stan, ${ }^{68}$ P. Stankus, ${ }^{94}$ P. J. Steffanic, ${ }^{130}$ E. Stenlund,${ }^{80}$ D. Stocco, ${ }^{114}$ M. M. Storetvedt, ${ }^{36}$ P. Strmen,${ }^{14}$ A. A. P. Suaide,${ }^{121}$ T. Sugitate, ${ }^{45}$ C. Suire, ${ }^{61}$ M. Suleymanov, ${ }^{15}$ M. Suljic, ${ }^{34}$ R. Sultanov, ${ }^{64}$ M. Šumbera, ${ }^{93}$ S. Sumowidagdo, ${ }^{50}$ K. Suzuki, ${ }^{113}$ S. Swain, ${ }^{66}$ A. Szabo, ${ }^{14}$ I. Szarka, ${ }^{14}$ U. Tabassam, ${ }^{15}$ G. Taillepied, ${ }^{134}$ J. Takahashi, ${ }^{122}$ G. J. Tambave, ${ }^{22}$ S. Tang, ${ }^{134,6}$ M. Tarhini, ${ }^{114}$ M. G. Tarzila, ${ }^{47}$ A. Tauro, ${ }^{34}$ G. Tejeda Muñoz, ${ }^{44}$ A. Telesca,${ }^{34}$ C. Terrevoli, ${ }^{126,29 a, 29 b}$ D. Thakur, ${ }^{49}$ S. Thakur, ${ }^{141}$ D. Thomas, ${ }^{119}$ F. Thoresen, ${ }^{88}$

R. Tieulent, ${ }^{135}$ A. Tikhonov, ${ }^{62}$ A. R. Timmins,${ }^{126}$ A. Toia,${ }^{69}$ N. Topilskaya,${ }^{62}$ M. Toppi,${ }^{51}$ F. Torales-Acosta, ${ }^{20}$ S. R. Torres ${ }^{120}$ S. Tripathy, ${ }^{49}$ T. Tripathy, ${ }^{48}$ S. Trogolo, ${ }^{26 a, 26 b, 29 a, 29 b}$ G. Trombetta ${ }^{33 a, 33 b}$ L. Tropp, ${ }^{38}$ V. Trubnikov, ${ }^{2}$ W. H. Trzaska, ${ }^{127}$ T. P. Trzcinski, ${ }^{142}$ B. A. Trzeciak, ${ }^{63}$ T. Tsuji, ${ }^{132}$ A. Tumkin, ${ }^{107}$ R. Turrisi, ${ }^{56}$ T. S. Tveter, ${ }^{21}$ K. Ullaland, ${ }^{22}$ E. N. Umaka, ${ }^{126}$ A. Uras, ${ }^{135}$ G. L. Usai, ${ }^{24 a, 24 b}$ A. Utrobicic, ${ }^{97}$ M. Vala, ${ }^{16,38}$ N. Valle, ${ }^{139}$ S. Vallero, ${ }^{58}$ N. van der Kolk, ${ }^{63}$ L. V. R. van Doremalen, ${ }^{63}$ M. van Leeuwen, ${ }^{63}$ P. Vande Vyvre, ${ }^{34}$ D. Varga, ${ }^{145}$ M. Varga-Kofarago, ${ }^{145}$ A. Vargas, ${ }^{44}$ M. Vargyas, ${ }^{127}$ R. Varma, ${ }^{48}$ M. Vasileiou, ${ }^{83}$ A. Vasiliev, ${ }^{87}$ O. Vázquez Doce, ${ }^{117,103}$ V. Vechernin, ${ }^{112}$ A. M. Veen ${ }^{63}$

E. Vercellin, ${ }^{26 a, 26 b}$ S. Vergara Limón, ${ }^{44}$ L. Vermunt, ${ }^{63}$ R. Vernet, ${ }^{7}$ R. Vértesi,${ }^{145}$ L. Vickovic, ${ }^{35}$ J. Viinikainen, ${ }^{127}$ Z. Vilakazi, ${ }^{131}$ O. Villalobos Baillie, ${ }^{109}$ A. Villatoro Tello, ${ }^{44}$ G. Vino, ${ }^{52}$ A. Vinogradov, ${ }^{87}$ T. Virgili,${ }^{30 a, 30 b}$ V. Vislavicius,${ }^{88}$ A. Vodopyanov, ${ }^{75}$ B. Volkel, ${ }^{34}$ M. A. Völkl, ${ }^{101}$ K. Voloshin, ${ }^{64}$ S. A. Voloshin, ${ }^{143}$ G. Volpe,${ }^{33 a, 33 b}$ B. von Haller, ${ }^{34}$ I. Vorobyev, ${ }^{103,117}$ D. Voscek, ${ }^{116}$ J. Vrláková, ${ }^{38}$ B. Wagner, ${ }^{22}$ Y. Watanabe, ${ }^{133}$ M. Weber, ${ }^{113}$ S. G. Weber, ${ }^{105}$ A. Wegrzynek, ${ }^{34}$ D. F. Weiser, ${ }^{102}$ S. C. Wenzel,,${ }^{34}$ J. P. Wessels, ${ }^{144}$ U. Westerhoff, ${ }^{144}$ A. M. Whitehead ${ }^{125}$ E. Widmann, ${ }^{113}$ J. Wiechula, ${ }^{69}$ J. Wikne, ${ }^{21}$ G. Wilk, ${ }^{84}$ J. Wilkinson, ${ }^{53}$ G. A. Willems, ${ }^{34}$ E. Willsher, ${ }^{109}$ B. Windelband, ${ }^{102}$ W. E. Witt, ${ }^{130}{ }^{\text {Y. Wu, }}{ }^{129}$ R. Xu, ${ }^{6}$ S. Yalcin, ${ }^{77}$ K. Yamakawa, ${ }^{45}$ S. Yang, ${ }^{22}$ S. Yano, ${ }^{137}$ Z. Yin, ${ }^{6}$ H. Yokoyama ${ }^{63}$ I.-K. Yoo, ${ }^{18}$ J. H. Yoon, ${ }^{60}$ S. Yuan, ${ }^{22}$ A. Yuncu, ${ }^{102}$ V. Yurchenko, ${ }^{2}$ V. Zaccolo, ${ }^{58,25 a, 25 b}$ A. Zaman, ${ }^{15}$ C. Zampolli, ${ }^{34}$ H. J. C. Zanoli, ${ }^{121}$ N. Zardoshti, ${ }^{34,109}$ A. Zarochentsev, ${ }^{112}$ P. Závada, ${ }^{67}$ N. Zaviyalov, ${ }^{107}$ H. Zbroszczyk, ${ }^{142}$ M. Zhalov, ${ }^{96}$ X. Zhang, ${ }^{6}$ Z. Zhang, ${ }^{6,134}$ C. Zhao, ${ }^{21}$ V. Zherebchevskii, ${ }^{112}$ N. Zhigareva, ${ }^{64}$ D. Zhou, ${ }^{6}$ Y. Zhou, ${ }^{88}$ Z. Zhou, ${ }^{22}$ J. Zhu, ${ }^{6}$ Y. Zhu, ${ }^{6}$ A. Zichichi, ${ }^{27 a, 27 b, 10}$ M. B. Zimmermann, ${ }^{34}$ G. Zinovjev, ${ }^{2}$ and N. Zurlo ${ }^{140}$

(A Large Ion Collider Experiment Collaboration)

\footnotetext{
${ }^{1}$ A.I. Alikhanyan National Science Laboratory (Yerevan Physics Institute) Foundation, Yerevan, Armenia

${ }^{2}$ Bogolyubov Institute for Theoretical Physics, National Academy of Sciences of Ukraine, Kiev, Ukraine

${ }^{3 a}$ Bose Institute, Department of Physics, Kolkata, India

${ }^{3 \mathrm{~b}}$ Centre for Astroparticle Physics and Space Science (CAPSS), Kolkata, India

${ }^{4}$ Budker Institute for Nuclear Physics, Novosibirsk, Russia

${ }^{5}$ California Polytechnic State University, San Luis Obispo, California, USA

${ }^{6}$ Central China Normal University, Wuhan, China

${ }^{7}$ Centre de Calcul de l'IN2P3, Villeurbanne, Lyon, France

${ }^{8}$ Centro de Aplicaciones Tecnológicas y Desarrollo Nuclear (CEADEN), Havana, Cuba

${ }^{9}$ Centro de Investigación y de Estudios Avanzados (CINVESTAV), Mexico City and Mérida, Mexico

${ }^{10}$ Centro Fermi-Museo Storico della Fisica e Centro Studi e Ricerche "Enrico Fermi', Rome, Italy
} 


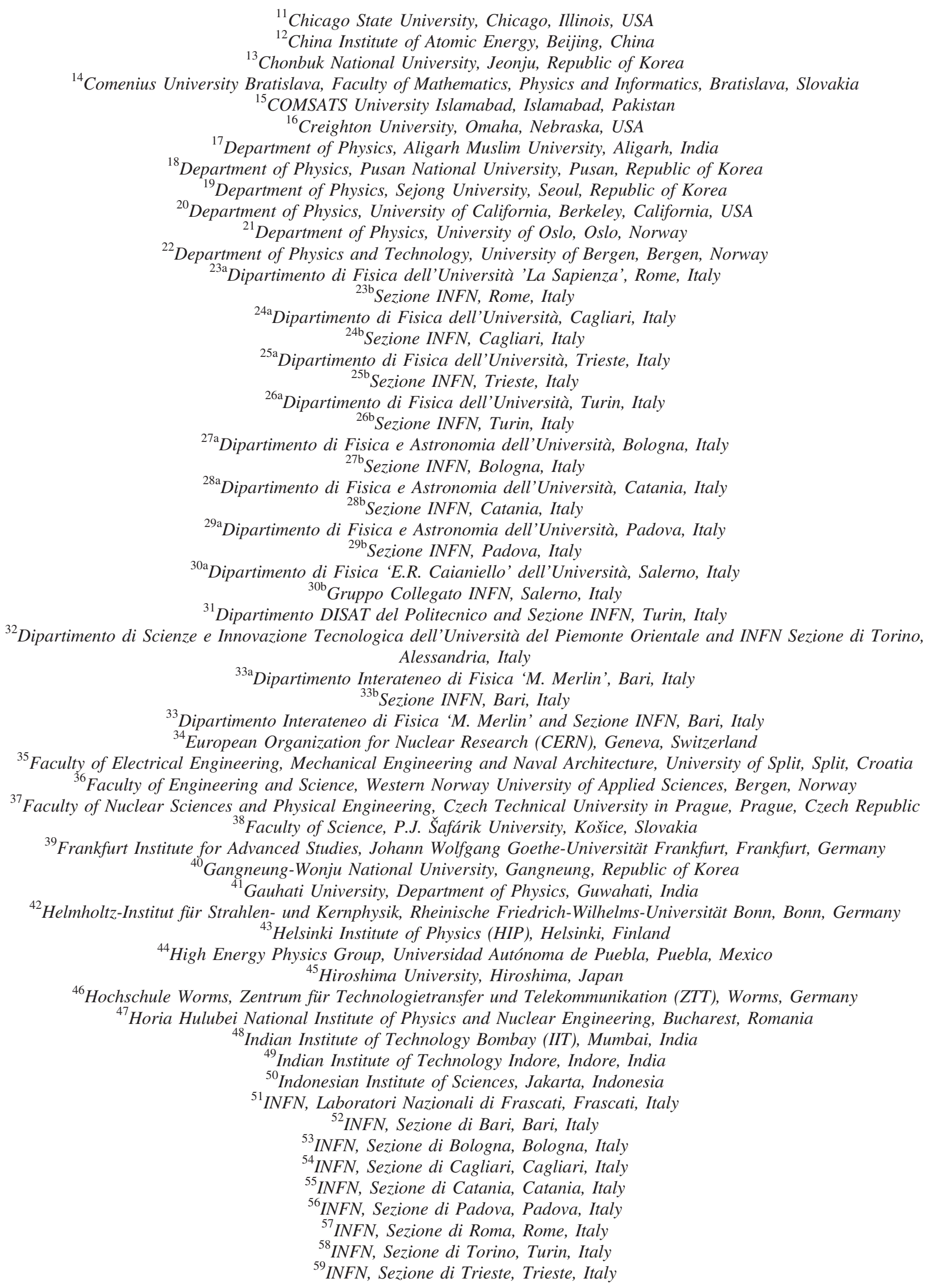


${ }^{60}$ Inha University, Incheon, Republic of Korea

${ }^{61}$ Institut de Physique Nucléaire d'Orsay (IPNO), Institut National de Physique Nucléaire et de Physique des Particules (IN2P3/CNRS), Université de Paris-Sud, Université Paris-Saclay, Orsay, France

${ }^{62}$ Institute for Nuclear Research, Academy of Sciences, Moscow, Russia

${ }^{63}$ Institute for Subatomic Physics, Utrecht University/Nikhef, Utrecht, Netherlands

${ }^{64}$ Institute for Theoretical and Experimental Physics, Moscow, Russia

${ }^{65}$ Institute of Experimental Physics, Slovak Academy of Sciences, Košice, Slovakia

${ }^{66}$ Institute of Physics, Homi Bhabha National Institute, Bhubaneswar, India

${ }^{67}$ Institute of Physics of the Czech Academy of Sciences, Prague, Czech Republic

${ }^{68}$ Institute of Space Science (ISS), Bucharest, Romania

${ }^{69}$ Institut für Kernphysik, Johann Wolfgang Goethe-Universität Frankfurt, Frankfurt, Germany

${ }^{70}$ Instituto de Ciencias Nucleares, Universidad Nacional Autónoma de México, Mexico City, Mexico

${ }^{71}$ Instituto de Física, Universidade Federal do Rio Grande do Sul (UFRGS), Porto Alegre, Brazil

${ }^{72}$ Instituto de Física, Universidad Nacional Autónoma de México, Mexico City, Mexico

${ }^{73}$ iThemba LABS, National Research Foundation, Somerset West, South Africa

${ }^{74}$ Johann-Wolfgang-Goethe Universität Frankfurt Institut für Informatik, Fachbereich Informatik und Mathematik, Frankfurt, Germany

${ }^{75}$ Joint Institute for Nuclear Research (JINR), Dubna, Russia

${ }^{76}$ Korea Institute of Science and Technology Information, Daejeon, Republic of Korea

${ }^{77}$ KTO Karatay University, Konya, Turkey

${ }^{78}$ Laboratoire de Physique Subatomique et de Cosmologie, Université Grenoble-Alpes, CNRS-IN2P3, Grenoble, France

${ }^{79}$ Lawrence Berkeley National Laboratory, Berkeley, California, USA

${ }^{80}$ Lund University Department of Physics, Division of Particle Physics, Lund, Sweden

${ }^{81}$ Nagasaki Institute of Applied Science, Nagasaki, Japan

${ }^{82}$ Nara Women's University (NWU), Nara, Japan

${ }^{83}$ National and Kapodistrian University of Athens, School of Science, Department of Physics, Athens, Greece

${ }^{84}$ National Centre for Nuclear Research, Warsaw, Poland

${ }^{85}$ National Institute of Science Education and Research, Homi Bhabha National Institute, Jatni, India

${ }^{86}$ National Nuclear Research Center, Baku, Azerbaijan

${ }^{87}$ National Research Centre Kurchatov Institute, Moscow, Russia

${ }^{88}$ Niels Bohr Institute, University of Copenhagen, Copenhagen, Denmark

${ }^{89}$ Nikhef, National institute for subatomic physics, Amsterdam, Netherlands

${ }^{90} \mathrm{NRC}$ Kurchatov Institute IHEP, Protvino, Russia

${ }^{91}$ NRNU Moscow Engineering Physics Institute, Moscow, Russia

${ }^{92}$ Nuclear Physics Group, STFC Daresbury Laboratory, Daresbury, United Kingdom

${ }^{93}$ Nuclear Physics Institute of the Czech Academy of Sciences, Řě̌ u Prahy, Czech Republic

${ }^{94}$ Oak Ridge National Laboratory, Oak Ridge, Tennessee, USA

${ }^{95}$ Ohio State University, Columbus, Ohio, USA

${ }^{96}$ Petersburg Nuclear Physics Institute, Gatchina, Russia

${ }^{97}$ Physics department, Faculty of science, University of Zagreb, Zagreb, Croatia

${ }^{98}$ Physics Department, Panjab University, Chandigarh, India

${ }^{99}$ Physics Department, University of Jammu, Jammu, India

${ }^{100}$ Physics Department, University of Rajasthan, Jaipur, India

${ }^{101}$ Physikalisches Institut, Eberhard-Karls-Universität Tübingen, Tübingen, Germany

${ }^{102}$ Physikalisches Institut, Ruprecht-Karls-Universität Heidelberg, Heidelberg, Germany

${ }^{103}$ Physik Department, Technische Universität München, Munich, Germany

${ }^{104}$ Politecnico di Bari, Bari, Italy

${ }^{105}$ Research Division and ExtreMe Matter Institute EMMI, GSI Helmholtzzentrum für Schwerionenforschung GmbH, Darmstadt, Germany

${ }^{106}$ Rudjer Bošković Institute, Zagreb, Croatia

${ }^{107}$ Russian Federal Nuclear Center (VNIIEF), Sarov, Russia

${ }^{108}$ Saha Institute of Nuclear Physics, Homi Bhabha National Institute, Kolkata, India

${ }^{109}$ School of Physics and Astronomy, University of Birmingham, Birmingham, United Kingdom

${ }^{110}$ Sección Física, Departamento de Ciencias, Pontificia Universidad Católica del Perú, Lima, Peru

${ }^{111}$ Shanghai Institute of Applied Physics, Shanghai, China

${ }^{112}$ St. Petersburg State University, St. Petersburg, Russia

${ }^{113}$ Stefan Meyer Institut für Subatomare Physik (SMI), Vienna, Austria

${ }^{114}$ SUBATECH, IMT Atlantique, Université de Nantes, CNRS-IN2P3, Nantes, France

${ }^{115}$ Suranaree University of Technology, Nakhon Ratchasima, Thailand

${ }^{116}$ Technical University of Košice, Košice, Slovakia

${ }^{117}$ Technische Universität München, Excellence Cluster 'Universe', Munich, Germany 
${ }^{118}$ The Henryk Niewodniczanski Institute of Nuclear Physics, Polish Academy of Sciences, Cracow, Poland

${ }^{119}$ The University of Texas at Austin, Austin, Texas, USA

${ }^{120}$ Universidad Autónoma de Sinaloa, Culiacán, Mexico

${ }^{121}$ Universidade de São Paulo (USP), São Paulo, Brazil

${ }^{122}$ Universidade Estadual de Campinas (UNICAMP), Campinas, Brazil

${ }^{123}$ Universidade Federal do ABC, Santo Andre, Brazil

${ }^{124}$ University College of Southeast Norway, Tonsberg, Norway

${ }^{125}$ University of Cape Town, Cape Town, South Africa

${ }^{126}$ University of Houston, Houston, Texas, USA

${ }^{127}$ University of Jyväskylä, Jyväskylä, Finland

${ }^{128}$ University of Liverpool, Liverpool, United Kingdom

${ }^{129}$ University of Science and Techonology of China, Hefei, China

${ }^{130}$ University of Tennessee, Knoxville, Tennessee, USA

${ }^{131}$ University of the Witwatersrand, Johannesburg, South Africa

${ }^{132}$ University of Tokyo, Tokyo, Japan

${ }^{133}$ University of Tsukuba, Tsukuba, Japan

${ }^{134}$ Université Clermont Auvergne, CNRS/IN2P3, LPC, Clermont-Ferrand, France

${ }^{135}$ Université de Lyon, Université Lyon 1, CNRS/IN2P3, IPN-Lyon, Villeurbanne, Lyon, France

${ }^{136}$ Université de Strasbourg, CNRS, IPHC UMR 7178, F-67000 Strasbourg, France, Strasbourg, France

${ }^{137}$ Université Paris-Saclay Centre d'Etudes de Saclay (CEA), IRFU, Départment de Physique Nucléaire (DPhN), Saclay, France

${ }^{138}$ Università degli Studi di Foggia, Foggia, Italy

${ }^{139}$ Università degli Studi di Pavia, Pavia, Italy

${ }^{140}$ Università di Brescia, Brescia, Italy

${ }^{141}$ Variable Energy Cyclotron Centre, Homi Bhabha National Institute, Kolkata, India

${ }^{142}$ Warsaw University of Technology, Warsaw, Poland

${ }^{143}$ Wayne State University, Detroit, Michigan, USA

${ }^{144}$ Westfälische Wilhelms-Universität Münster, Institut für Kernphysik, Münster, Germany

${ }^{145}$ Wigner Research Centre for Physics, Hungarian Academy of Sciences, Budapest, Hungary

${ }^{146}$ Yale University, New Haven, Connecticut, USA

${ }^{147}$ Yonsei University, Seoul, Republic of Korea

${ }^{\mathrm{a}}$ Deceased.

${ }^{\mathrm{b}}$ Also at Dipartimento DET del Politecnico di Torino, Turin, Italy.

${ }^{c}$ Also at M.V. Lomonosov Moscow State University, D.V. Skobeltsyn Institute of Nuclear, Physics, Moscow, Russia.

${ }^{\mathrm{d}}$ Also at Department of Applied Physics, Aligarh Muslim University, Aligarh, India.

${ }^{\mathrm{e}}$ Also at Institute of Theoretical Physics, University of Wroclaw, Polan. 